\title{
Integrated Guidance and Control Based Air-to-Air Autonomous Attack Occupation of UCAV
}

\author{
Chang Luo, ${ }^{1}$ Jie Wang, ${ }^{1}$ Hanqiao Huang, ${ }^{2}$ and Pengfei Wang ${ }^{1}$ \\ ${ }^{1}$ Air and Missile Defense College, Air Force Engineering University, Xian 710051, China \\ ${ }^{2}$ Aeronautics and Astronautics Engineering College, Air Force Engineering University, Xian 710038, China \\ Correspondence should be addressed to Chang Luo; luochang1988@126.com
}

Received 19 April 2016; Revised 22 July 2016; Accepted 14 August 2016

Academic Editor: Kalyana C. Veluvolu

Copyright (c) 2016 Chang Luo et al. This is an open access article distributed under the Creative Commons Attribution License, which permits unrestricted use, distribution, and reproduction in any medium, provided the original work is properly cited.

\begin{abstract}
An approach of air-to-air autonomous attack occupation for Unmanned Combat Aerial Vehicles (UCAVs) is proposed to improve attack precision and combat effectiveness. According to the shortage of UCAV in the task of attack occupation, kinematic and dynamic models of UCAV and missile loaded on it are formed. Then, attack zone and no-escape zone are calculated by pattern search algorithm, and the optimum attack position is indicated. To arrive at the optimum attack position accurately with restriction of gesture, a novel adaptive sliding mode control method is suggested to design the integrated guidance and control system of UCAV in the process of autonomous attack occupation. Key parameters of the control system are adaptively regulated, which further economize control energy at the same time. The simulation results show that compared with traditional methods our approach can guide the UCAV to the optimum attack position with stable gesture and economize nearly $25 \%$ control energy.
\end{abstract}

\section{Introduction}

In most UCAV systems (e.g., Predator. MQ-1B), operator on the ground is a key component of the whole control loop. However, the connatural limitation of operator brings drawback of the UCAV system by limiting the precision of estimation and time of response. In fact, the UCAV and operator are in different frame of time-space. To solve this problem, technology of autonomous attack for UCAV has become a hot topic in recent years [1-5]. Yet, many achievements of researchers are aimed at attacking ground fixed targets [2-4]. Technologies of air-to-air autonomous attack occupation for UCAV still remain as a novel field [5].

Air-to-air autonomous attack occupation of UCAV is mainly comprised of two relative processes. Firstly, after having detected target in the air, calculating the optimal position that UCAV should maneuver to is crucial for the follow-up processes. As to aerial maneuvering target, Zhang et al. [6] calculate the real-time attack zone with golden section method. However, the maneuver capability of UCAV is relatively poor for its high-aspect-ratio, and its attack zone is relatively small. Compared with the timeliness, the precision of attack occupation is more important [7]. In [8], a dynamic attack zone is calculated, which can be reliably and accurately used during any flight antagonism time. But, for autonomous attack occupation of UCAV, more factors should be taken into consideration, such as the no-escape zone and optimum attack position [1,9]. Secondly, after having attained the optimum attack position, UCAV should maneuver to it accurately and rapidly. Integrated guidance and control (IGC) can eliminate the time-lag that inevitably exists in the conventional guidance and control design that separates the guidance loop from the control loop [10-12]. In addition, IGC improves the precision of the terminal phase of guidance [13-15]. Inspired by this, we propose combining IGC with the maneuver process of attack occupation of UCAV. Many researches have confirmed the effectiveness of IGC in various guidance and control scenarios [16-20]. In [19], high order sliding mode control (SMC) approach is incorporated with IGC to design the interception of maneuvering hypersonic vehicle. Guo and Liang [20] design an IGC system based on the block backstepping sliding mode and extended state observer for the near-space interceptor. However, most researches above are based on the scenario 
in which the maneuver of target could be estimated exactly. For the scenario of autonomous attack occupation of UCAV, more attention should be placed on automaticity and adaptability.

Therefore, motivated by the aforementioned considerations we compute the attack zone and no-escape zone of UCAV with pattern search algorithm, which is famous for its precision. Then, by combining the practicality of air combat to the no-escape zone computed before, we attain the optimum attack position. Furthermore, a novel adaptive SMC method is used to design the IGC system of UCAV during the process of maneuvering to the optimum attack position. The proposed control system satisfies the gesture constraints of UCAV at the attack moment. In addition, the key parameters of the control system are timely and adaptively regulated in the attack occupying process, which brings better performance and less consumption of control energy in the simulation scenario.

Our main contribution is that we design the air-toair autonomous attack occupation of UCAV based on the key factors of air combat. After analyzing the process of autonomous attack occupation theoretically and practically, we compute the optimum attack position. Then, we combine a novel adaptive SMC method to the IGC system of UCAV, so that the UCAV can maneuver to the optimum attack position rapidly and accurately and satisfy the gesture constraints at the attack moment. To our knowledge, there have not been any attempts of applying IGC approach to UCAV in the airto-air autonomous attack occupation.

The rest of the paper is organized as follows. In Section 2, the task and kinematic model of air-to-air autonomous attack are derived. Section 3 introduces the computation of attack zone, no-escape zone, and optimum attack position, which provides the target position that the UCAV should maneuver to with our proposed integrated guidance and control approach explained in Section 4. Numerical simulations are demonstrated in Section 5 and we conclude the paper in Section 6.

\section{Air-to-Air Autonomous Attack Occupation of UCAV}

2.1. Air-to-Air Autonomous Attack Task of UCAV. Air-toair autonomous attack task for UCAV can generally be divided into four phases: takeoff and cruise, target state estimation and attack zone calculation, autonomous attack occupying, and weapon launching. Four phases correspond to $\overparen{A_{1} B}\left(\widehat{A_{2} B}\right), \overparen{B C}, \overparen{C D}$, and $\overparen{D E}$, respectively in Figure 1 .

In the phase of takeoff and cruise, UCAV fulfills path planning and real-time preplanning according to the battlefield environment and mission. When airborne detection system finds aerial target, the tracking system provides target information to the integrated fire and flight (IFF) computer. The computer estimates target state and computes the attack zone. Then according to the condition of the guided missile, the range of attack zone, and the threat from target, UCAV is guided to the optimum attack position. When the weapon management system meets the need of launch conditions, UCAV launches the guided missile.
2.2. Kinematic Modeling of Air-to-Air Attack Task. UCAV fulfills air-to-air autonomous attack in three-dimensional space. The relative movement of UCAV and the target can be separately studied in the vertical and lateral plane, as is shown in Figure 2.

$\lambda_{P}$ and $\lambda_{L}$ denote the line-of-sight (LOS) angle in vertical and lateral planes, respectively.

In the vertical plane, the relative movement of UCAV and the target is shown in Figure 3. $R_{P}$ is the relative distance between them. $V_{U P}, a_{U P}$, and $\theta_{U P}$ are the velocity, normal acceleration, and flight-path angle of UCAV, respectively. The velocity, normal acceleration, and path angle of target are denoted by $V_{T P}, a_{T P}$, and $\theta_{T P}$ respectively.

The relative movement of UCAV and the target shown in Figure 3 can be described by

$$
\begin{aligned}
\dot{R}_{P} & =V_{T P} \cos \left(\theta_{T P}-\lambda_{P}\right)-V_{U P} \cos \left(\theta_{U P}-\lambda_{P}\right), \\
R_{P} \dot{\lambda}_{P} & =V_{T P} \sin \left(\theta_{T P}-\lambda_{P}\right)-V_{U P} \sin \left(\theta_{U P}-\lambda_{P}\right), \\
\dot{\theta}_{U P} & =\frac{a_{U P}}{V_{U P}}, \\
\dot{\theta}_{T P} & =\frac{a_{T P}}{V_{T P}} .
\end{aligned}
$$

Similar to (1), the relative movement in lateral plane is given as follows:

$$
\begin{aligned}
\dot{R}_{L} & =V_{T L} \cos \left(\theta_{T L}-\lambda_{L}\right)-V_{U L} \cos \left(\theta_{U L}-\lambda_{L}\right), \\
R_{L} \dot{\lambda}_{L} & =V_{T L} \sin \left(\theta_{T L}-\lambda_{L}\right)-V_{U L} \sin \left(\theta_{U L}-\lambda_{L}\right), \\
\dot{\theta}_{U L} & =\frac{a_{U L}}{V_{U L}}, \\
\dot{\theta}_{T L} & =\frac{a_{T L}}{V_{T L}} .
\end{aligned}
$$

In this paper, the guided missile is axisymmetric and irrotational. Because the calculation of attack zone is a process of generating trajectory substantially, the guided missile is modeled in three degrees of freedom:

$$
\begin{aligned}
& \dot{x}_{m}=v_{m} \cos \theta_{m} \cos \varphi_{m}, \\
& \dot{y}_{m}=v_{m} \cos \theta_{m} \sin \varphi_{m}, \\
& \dot{h}_{m}=v_{m} \sin \theta_{m}, \\
& \dot{v}_{m}=\frac{\left(P_{m}-D_{m}\right)}{m_{m}}-g \sin \theta_{m}, \\
& \dot{\theta}_{m}=\frac{\left(n_{m y}-\cos \theta_{m}\right) g}{v_{m}}, \\
& \dot{\varphi}_{m}=\frac{n_{m z} g}{v_{m} \cos \theta_{m}},
\end{aligned}
$$

where $\left(x_{m}, y_{m}, h_{m}\right)$ denotes the missile's position. $v_{m}, \theta_{m}$, $\varphi_{m}$ denote missile's velocity, path angle, and azimuth angle, respectively. $P_{m}$ is the thrust of the missile, and $D_{m}$ is the 


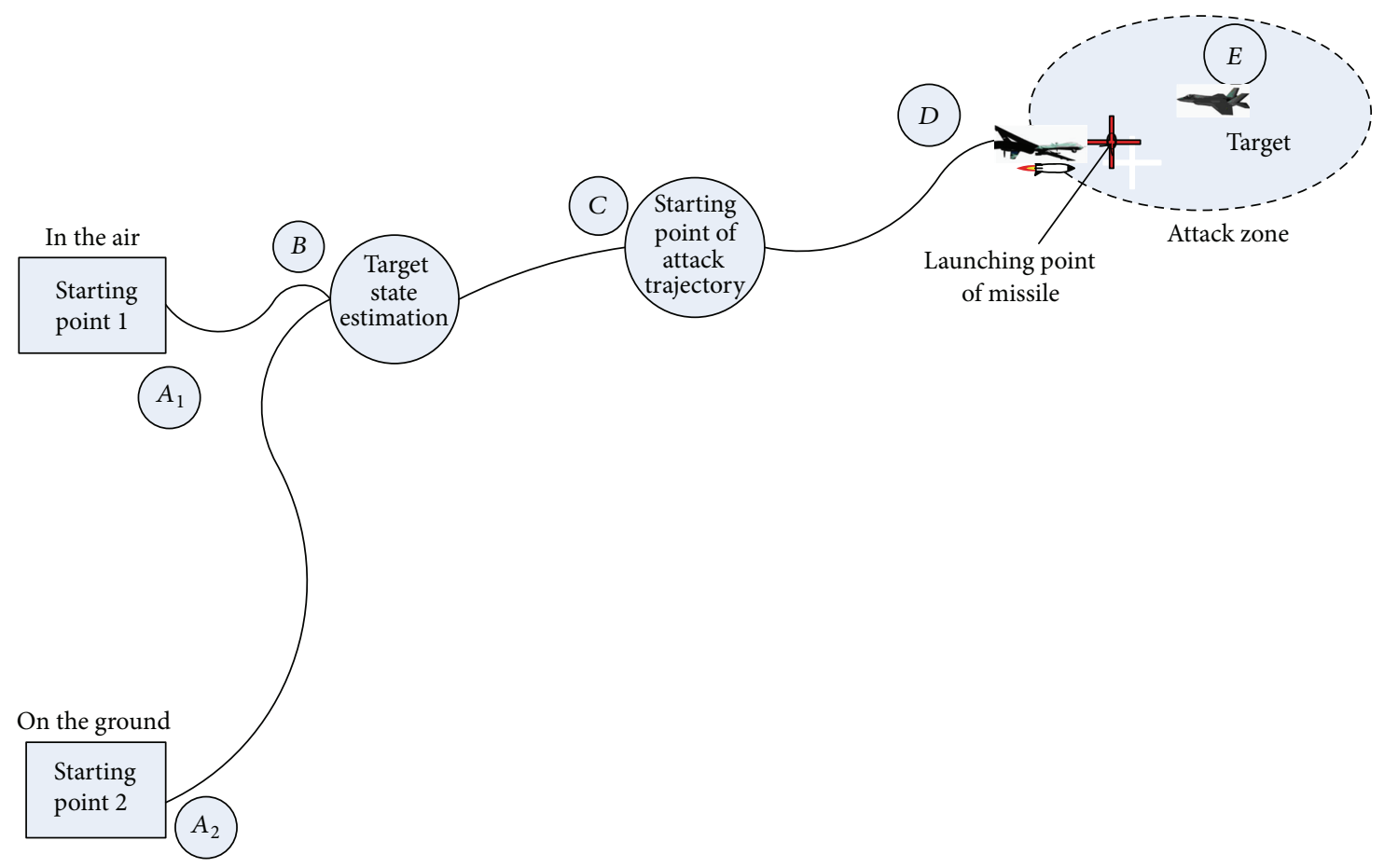

Figure 1: Assignment of air-to-air autonomous attack.

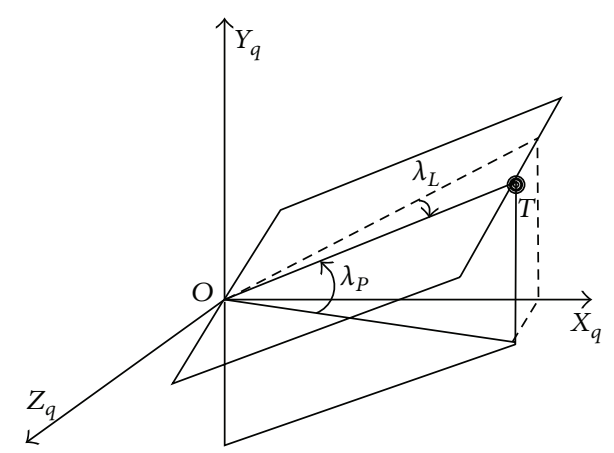

FIGURE 2: Kinematic relation of UCAV and the target.

drag force of it. $m_{m}$ is the mass of the missile. $n_{m y}$ and $n_{m z}$ are the $g$-load of the missile in pitching and yawing direction, respectively.

\section{The Optimum Attack Position}

3.1. Calculation of Attack Zone. During the implementation of autonomous attack task, UCAV faces up to corresponding threat from the target. Concerning the properties of UCAV, such as abundant useable $g$-load, relatively small payload, and small attack zone, the precision of attack zone becomes essential. We compute the attack zone with the pattern search algorithm for higher precision [21-24]. Pattern search algorithm includes two types of moves: (i) exploratory move, which is made on current point by investigation along each independent search direction, and (ii) pattern move, which finds the new point by jumping from present base point

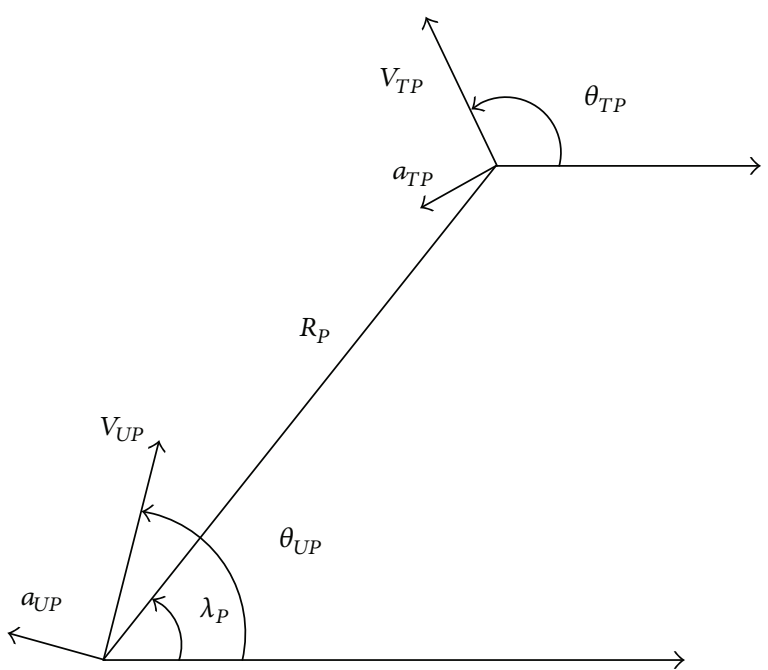

FIGURE 3: Kinematic relation of UCAV and the target in vertical plane.

along with pattern direction [25]. The two types of moves are alternated to find the boundary of attack distance. Pattern search algorithm provides global optimal results without derivatives of objective functions [26], and it is further illustrated in Figure 4.

In Figure 4, search directions along the axes at points $x^{(k)}$ and $x^{(k+1)}$ indicate exploratory move, which are drawn as solid lines with arrowheads. On the other hand, pattern direction along the broken line from $x^{(k)}$ to $x^{(k+1)}$ indicates pattern move. Furthermore, we introduce a contraction parameter 
(1) Set initial state $x^{(0)}$, initial step size $\delta^{0}=\left(\delta_{1}{ }^{0}, \delta_{2}{ }^{0}, \ldots, \delta_{n}{ }^{0}\right)^{T}$, acceleration parameter $\gamma>1$, contraction parameter $\eta \in(0,1)$ and required accuracy $\varepsilon>0$.

(2) Let $y=x^{(k)}$.

(3) Apply the exploratory move in the direction that parallel to the unit vector $e^{j}(j=1,2, \ldots, n)$.

(4) if $f\left(y+\delta_{j}{ }^{k} e^{j}\right)<f(y)$ then $y=y+\delta_{j}{ }^{k} e^{j}$

(5) else if $f\left(y-\delta_{j}{ }^{k} e^{j}\right) \leq f(y)$ then $y=y-\delta_{j}{ }^{k} e^{j}$

(6) else $y=y$

(7) end if.

(8) Let $x^{(k+1)}=y$.

(9) if $f\left(x^{(k+1)}\right)<f\left(x^{(k)}\right)$ then apply the pattern move $y=x^{(k+1)}+\gamma\left(x^{(k+1)}-x^{(k)}\right)$, set $k=k+1$ and go to (3)

(10) else go to (12)

(11) end if.

(12) if $\left|\delta^{k}\right|<\varepsilon$ then stop iteration and output $x^{(k)}$

(13) else set $y=x^{(k+1)}, \delta^{k+1}=\eta \delta^{k}, k=k+1$ and go to (3)

(14) end if.

Algorithm 1: Pattern search algorithm with variable step size.

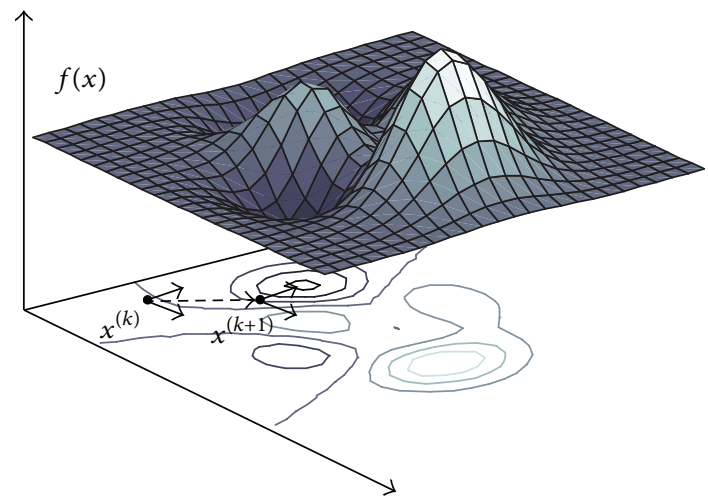

FIGURE 4: Diagram of pattern search algorithm.

$\eta \in(0,1)$ to the traditional pattern search algorithm. The contraction parameter can change the size of search step to speed up search process at the initial phase as well as improve the search accuracy at the terminal phase. Suppose $f(x)$ is the distance between UCAV and the target in accordance with all the constraints. Set the iterative number $k=0$, and we do as shown in Algorithm 1.

The pattern search algorithm above is used to calculate the near boundary of attack distance. The far boundary of attack distance can be obtained by replacing the object function $f(x)$ with $-f(x)$.

The calculation flow of attack distance can be further shown in Figure 5.

The process of calculating attack zone involves the inner loop and outer loop. The inner loop is about to search the maximum or minimum distance in a certain entry angle with respect to the target. The outer loop searches through the entry angles, whose bound is $[0,2 \pi]$. Then, attack zone can be calculated by means of interpolating or fitting method.

The outer loop of attack zone calculation is presented in Figure 6.

For the missile loaded on UCAV, assume that its offaxis angle is 0 , initial weight is $22 \mathrm{~kg}$, initial velocity $v_{m 0}$
$=300 \mathrm{~m} / \mathrm{s}$, consumption of fuel per second $c_{m}=1.5 \mathrm{~kg} / \mathrm{s}$, working time of engine $t_{0}=4 \mathrm{~s}$, average thrust $\bar{P}=1000 \mathrm{~N}$, delay time of actuator $\tau=0.02 \mathrm{~s}$, maximum LOS angle $q_{\max }$ $=60^{\circ}$, maximum tracking rate of LOS angle $\omega_{\max }=20^{\circ} / \mathrm{s}$, available $g$-load $n_{K}=20 \mathrm{~g}$, unlock time of fuze $t_{d}=0.5 \mathrm{~s}$, and maximum available distance of fuze is $50 \mathrm{~m}$. At the same time, assume that the target flies with no lateral $g$-load and its velocity $V_{T}=300 \mathrm{~m} / \mathrm{s}$. Considering the combat height $H=$ $6 \mathrm{~km}$, the attack zone calculated by pattern search algorithm is illustrated in Figure 7.

As shown in Figure 7, the far boundary of attack zone becomes larger with the increase of entry angle $\sigma$, and it reaches the maximum when entry angle $\sigma=180^{\circ}$. Then, it gradually shrinks. This is consistent with our intuition that the far boundary of attack zone is mainly affected by the approaching speed of the missile and target. The missile can reach the target in a relatively far distance when it is launched in front of the target. On the contrary, the attack distance decreases when the missile is launched behind the target. On the other hand, the near boundary of attack zone is mainly affected by the approaching speed of them within the work time of fuze on the missile. The variation trend of near boundary is the same as that of far boundary.

3.2. Calculation of No-Escape Zone. The practice of air combat shows that launching missile within the attack zone cannot guarantee destroying the target when it implements large overload maneuver. Thus, after calculating the attack zone, we further investigate the attack zone in two other cases, in which the aerial target maneuvers in the left direction and right direction with maximum $g$-load. Then the noescape zone is attained by choosing the minimum of the three far boundaries of attack zone as the far boundary and the maximum of the three near boundaries of attack zone as the near boundary:

$$
\begin{aligned}
& R_{\max }^{\text {no-escape }} \\
& \quad=\min \left\{R_{\max }\left(n_{T \max }\right), R_{\max }\left(-n_{T \max }\right), R_{\max }(0)\right\},
\end{aligned}
$$




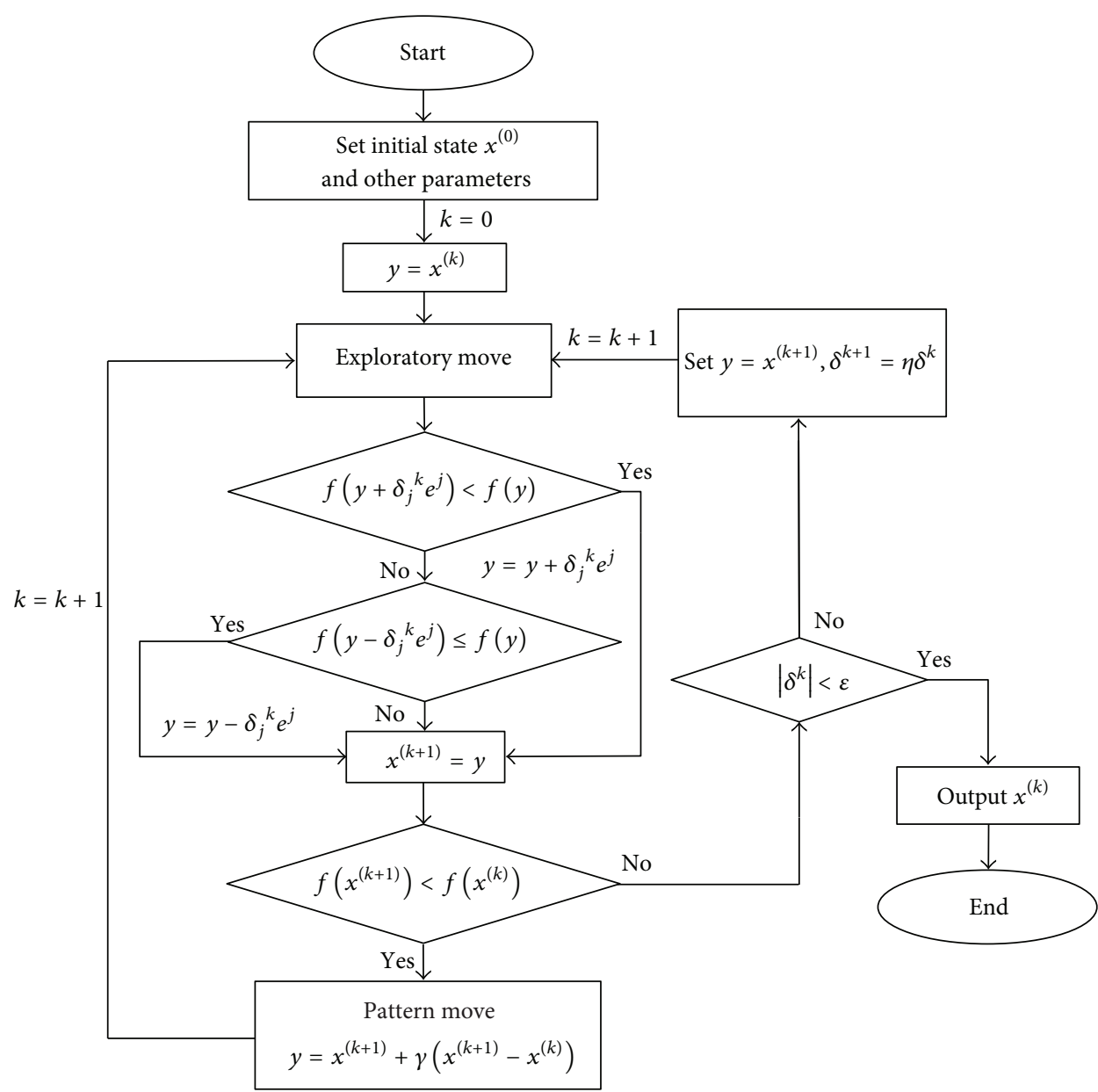

FIGURE 5: Flowchart of pattern search algorithm for calculating attack distance.
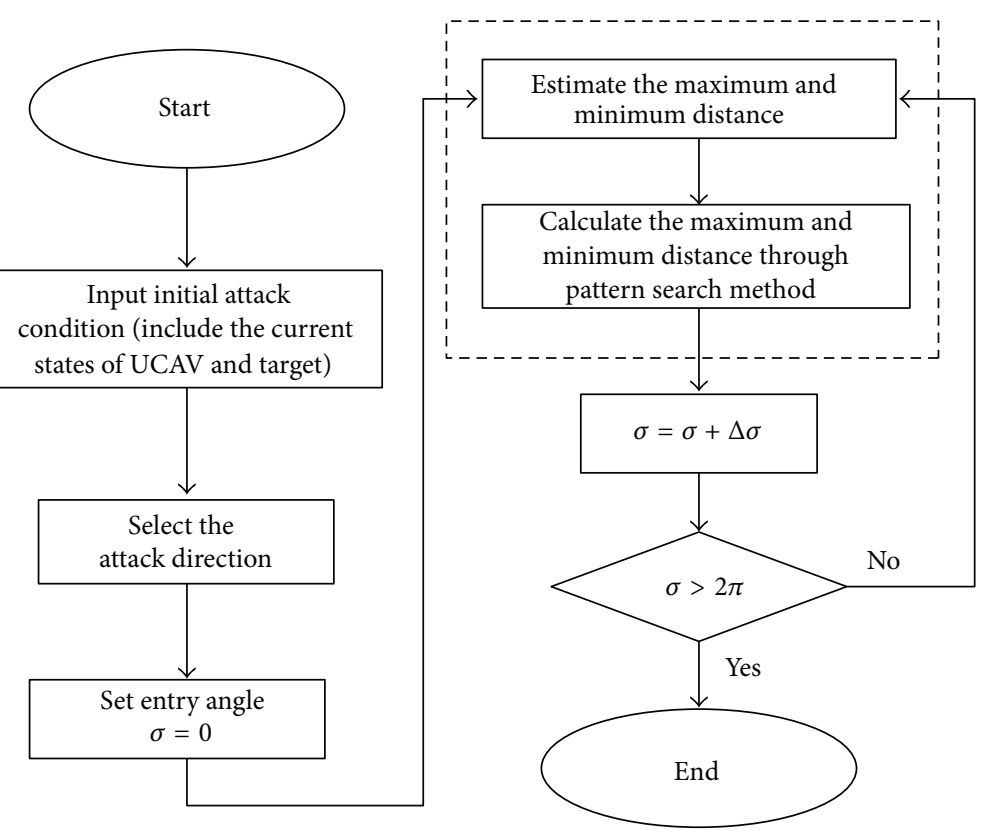

FIGURE 6: Flowchart of calculating the attack zone. 


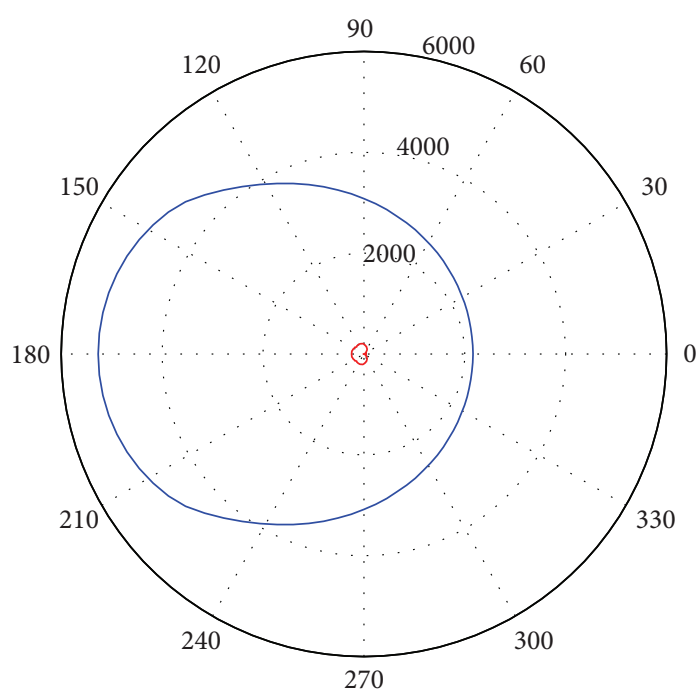

FIGURE 7: Attack zone.

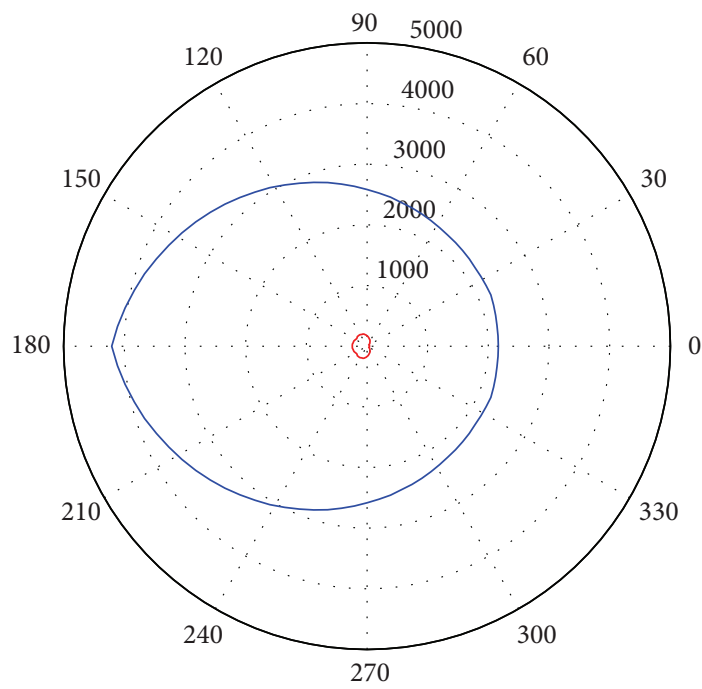

Figure 8: No-escape zone.

$$
\begin{aligned}
& R_{\text {min }}^{\text {no-escape }} \\
& \quad=\max \left\{R_{\min }\left(n_{T \max }\right), R_{\min }\left(-n_{T \max }\right), R_{\min }(0)\right\} .
\end{aligned}
$$

Assuming the maximum $g$-load of target $\left|n_{T \max }\right|=3 g$, the calculated no-escape zone is shown in Figure 8.

Compared with the attack zone in Figure 7 , the far boundary of no-escape zone in Figure 8 shrinks. In the case of launching missile in front of the target, the maximum attack distance decreases about $1 \mathrm{~km}$. On the other hand, the near boundary of no-escape zone becomes a little larger than that of attack zone in Figure 7.

3.3. Optimum Attack Position for UCAV. Combining the practicality of air combat to the implementation of air-toair autonomous attack, UCAV should satisfy the following qualifications:

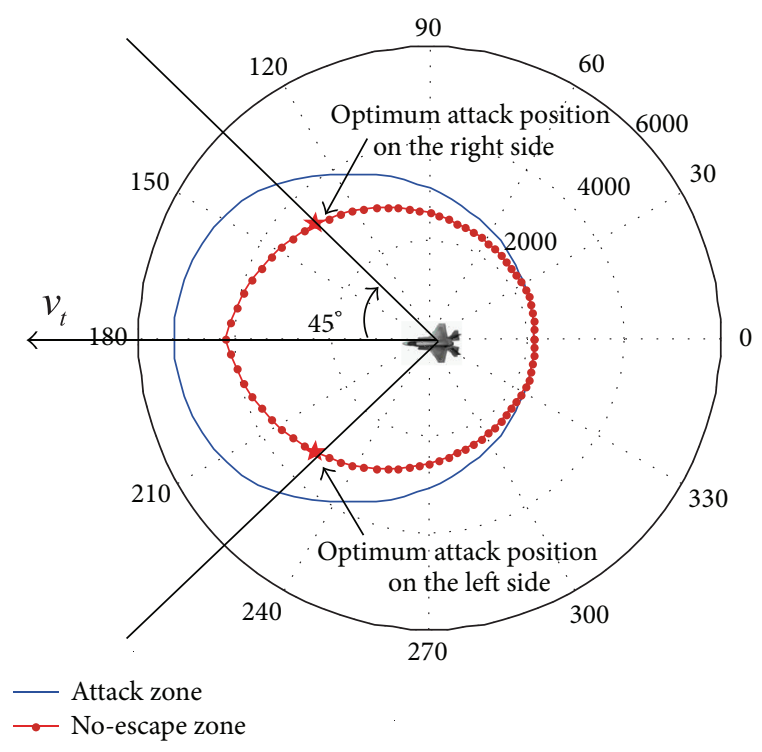

FIgURE 9: The optimum attack position of UCAV.

(1) Keep away from the detection range of fire-control radar that is loaded on the target.

(2) Considering the target's maneuver ability, the probability of destroying it by guided missile should be as high as possible.

(3) Distance between UCAV and the target should be as far as possible, and, before being discovered by the target, attack first.

Assume that the range of the azimuth angle of target's fire-control radar is $\pm 45^{\circ}$. Considering the calculating result of attack zone and no-escape zone, as well as the specified qualifications above, the optimum attack position of UCAV can be illustrated in Figure 9.

Figure 9 shows two optimum attack positions of the UCAV relative to the left and right direction of the target. Both attack positions are on the far boundary of the no-escape zone. When UCAV launches missile at these two positions, no matter how the target maneuvers at the combat height the launched missile can destroy it. In addition, these two points are both on the boundary of the range that the target's firecontrol radar can reach. The probability of being detected by target is relatively low in this case. Finally, the choice of the specific position depends on the UCAV's azimuth relative to the target when UCAV detects it.

\section{Autonomous Attack Occupation Based on Integrated Guidance and Control}

Before autonomous attack occupation, UCAV usually makes full use of its stealth characteristics and gets close to the enemy aircraft with negative height. Then it maneuvers to the optimum attack position and launches the guided missile. Figure 10 describes the whole process. 


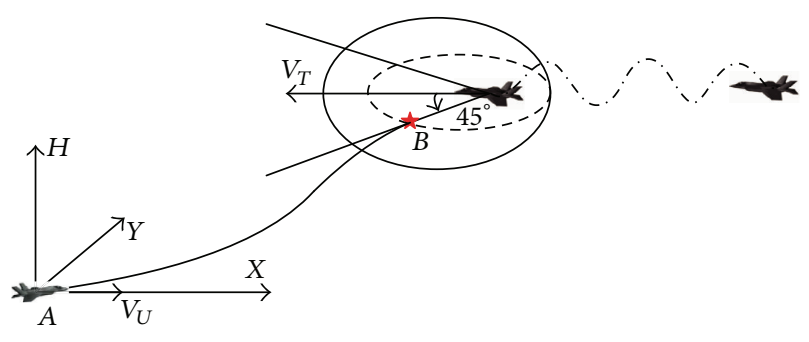

FIGURE 10: Diagram of guide process of UCAV.

To achieve the process of autonomous attack occupation rapidly and accurately, integrated guidance and control system is designed, respectively, for pitch channel, yaw channel, and roll channel of the UCAV.

4.1. Gesture Constraints at the Attack Moment. When launching the guided missile, the constraints of UCAV's gesture are substantially about constraints of pitch angle $\vartheta$ and yaw angle $\psi$. In the terminal phase of guidance, the angle of attack $\alpha \approx 0$, the angle of sideslip $\beta \approx 0, \vartheta=\theta_{U P}+\alpha$, and $\psi=\theta_{U L}+\beta$. Define $\vartheta_{q}$ and $\psi_{q}$ as the pitch angle and yaw angle of the UCAV at the attack moment. The gesture constraints can be described as

$$
\begin{gathered}
\left.\vartheta_{q} \approx \theta_{U P}\right|_{t_{g o}=0}=\theta_{q p}, \\
\left.R_{P}\right|_{t_{g o}=0}=0, \\
\left.\psi_{q} \approx \theta_{U L}\right|_{t_{g o}=0}=\theta_{q l}, \\
\left.R_{L}\right|_{t_{g o}=0}=0 .
\end{gathered}
$$

According to conclusion in Section 3.3, which is illustrated in Figure 9, we can obtain

$$
\begin{aligned}
& \theta_{q p}=0, \\
& \theta_{q l}=\theta_{t q l} \pm 135^{\circ},
\end{aligned}
$$

where $\theta_{t q l}$ is the azimuth angle of UCAV with respect to the enemy aircraft at the attack moment.

During the process of autonomous attack occupation, the UCAV should meet the requirements that the $g$-load of it may be as small as possible and the maneuver path of it may be as straight as possible. That is to say, we hope that the UCAV can be guided with its LOS angular rate approaching 0 as soon as possible, and it can be described as follows:

$$
\begin{aligned}
& \dot{\lambda}_{P}=0, \\
& \dot{\lambda}_{L}=0 .
\end{aligned}
$$

Then, the terminal LOS angle can be described as

$$
\begin{aligned}
& \lambda_{q p}=\theta_{q p}=0, \\
& \lambda_{q l} \\
& =\tan ^{-1} \frac{\left(V_{T L}+(\sqrt{2} / 2) V_{U L}\right) \sin \theta_{t q l}-(\sqrt{2} / 2) V_{U L} \cos \theta_{t q l}}{\left(V_{T L}+(\sqrt{2} / 2) V_{U L}\right) \cos \theta_{t q l}+(\sqrt{2} / 2) V_{U L} \sin \theta_{t q l}}
\end{aligned}
$$

$$
\begin{aligned}
& \lambda_{q p}=\theta_{q p}=0, \\
& \lambda_{q l} \\
& =\tan ^{-1} \frac{\left(V_{T L}+(\sqrt{2} / 2) V_{U L}\right) \sin \theta_{t q l}+(\sqrt{2} / 2) V_{U L} \cos \theta_{t q l}}{\left(V_{T L}+(\sqrt{2} / 2) V_{U L}\right) \cos \theta_{t q l}-(\sqrt{2} / 2) V_{U L} \sin \theta_{t q l}} .
\end{aligned}
$$

Equations (8) and (9), respectively, describe the gesture constraints on condition that the UCAV implements autonomous attack occupation from the left and right directions with relativity to the target.

\subsection{Modeling and Standardizing the IGC System of UCAV}

4.2.1. Pitch Channel. According to the relative movement between UCAV and the target described by (1),

$$
\begin{aligned}
\ddot{\lambda}_{P}= & -\frac{2 \dot{R}_{P}}{R_{P}} \dot{\lambda}_{P}+\frac{\cos \left(\theta_{T P}-\lambda_{P}\right)}{R_{P}} a_{T P} \\
& -\frac{\cos \left(\theta_{U P}-\lambda_{P}\right)}{R_{P}} a_{U P} .
\end{aligned}
$$

And in the terminal phase of attack occupation

$$
\lambda_{P} \approx \theta_{U P}
$$

So $\ddot{\lambda}_{P}$ can be further described as

$$
\ddot{\lambda}_{P}=-\frac{2 \dot{R}_{P}}{R_{P}} \dot{\lambda}_{P}-\frac{a_{U P}^{\prime}}{R_{P}}+\frac{\cos \left(\theta_{T P}-\lambda_{P}\right)}{R_{P}} a_{T P},
$$

where $a_{U P}^{\prime}=\cos \left(\theta_{U P}-\lambda_{P}\right) a_{U P} \approx a_{U P}$. What is more, because the target's acceleration is small before it has detected the UCAV, we could consider its acceleration as an unknown bounded variable. Let $d_{\lambda_{P 1}}=\cos \left(\theta_{T P}-\lambda_{P}\right) a_{T P}$, and (12) becomes

$$
\ddot{\lambda}_{P}=-\frac{2 \dot{R}_{P}}{R_{P}} \dot{\lambda}_{P}-\frac{a_{U P}^{\prime}}{R_{P}}+\frac{d_{\lambda_{P 1}}}{R_{P}} .
$$

Because of the high-aspect-ratio structure, lateral force caused by sideslip angle has little effect on the UCAV, and hence

$$
a_{U P}^{\prime} \approx \frac{\left(\rho V_{U P}^{2} S c_{y}^{\alpha}+2 P\right) \alpha \cos \gamma}{2 m}+d_{a_{U P}},
$$

where $S, P, \gamma$, and $m$ are wing area, engine thrust, roll angle, and mass of the UCAV, respectively. $\rho$ denotes the atmospheric density and $c_{y}^{\alpha}$ is an aerodynamic constant and $d_{a_{U P}}$ denotes the approximate error of lateral acceleration in the vertical plane. In this case, (13) can be substituted by

$$
\begin{aligned}
\ddot{\lambda}_{P}= & -\frac{2 \dot{R}_{P}}{R_{P}} \dot{\lambda}_{P}-\frac{\left(\rho V_{U P}^{2} S c_{y}^{\alpha}+2 P\right) \alpha \cos \gamma}{2 m R_{P}} \\
& +\frac{d_{\lambda_{P 1}}-d_{a_{U P}}}{R_{P}} \\
= & -\frac{2 \dot{R}_{P}}{R_{P}} \dot{\lambda}_{P}-\frac{\left(\rho V_{U P}^{2} S c_{y}^{\alpha}+2 P\right) \alpha \cos \gamma}{2 m R_{P}}+d_{\lambda_{P}} .
\end{aligned}
$$


In (15), $d_{\lambda_{P}}$ denotes an unknown bounded variable, which contains the approximate error of lateral acceleration and the unknown information of target's maneuver.

According to the gesture constraints analyzed in Section 4.1, UCAV's angle of attack is small when it arrives at the optimum attack position.

So, at this moment

$$
\lambda_{q p} \approx \vartheta_{q}=\theta_{q p} .
$$

Then, the state variables, control variable, and output variables can be described as follows:

$$
\begin{aligned}
& \mathbf{x}=\left[x_{1}, x_{2}, x_{3}, x_{4}\right]^{T}=\left[\lambda_{P}-\lambda_{q p}, \dot{\lambda}_{P}, \alpha, \omega_{z}\right]^{T}, \\
& u=\delta_{e}, \\
& \mathbf{y}=\left[x_{3}, x_{4}\right]^{T}=\left[\alpha, \omega_{z}\right]^{T} .
\end{aligned}
$$

Combining (17) with (15) and considering the coupling between the pitch channel and other channels as unknown bounded variable, we can obtain the IGC model of pitch channel as follows:

$$
\begin{aligned}
{\left[\begin{array}{l}
\dot{x}_{1} \\
\dot{x}_{2} \\
\dot{x}_{3} \\
\dot{x}_{4}
\end{array}\right] } & =\left[\begin{array}{cccc}
0 & 1 & 0 & 0 \\
0 & a_{22} & a_{23} & 0 \\
0 & 0 & a_{33} & 1 \\
0 & 0 & a_{43} & 0
\end{array}\right]\left[\begin{array}{l}
x_{1} \\
x_{2} \\
x_{3} \\
x_{4}
\end{array}\right]+\left[\begin{array}{l}
0 \\
0 \\
0 \\
b_{4}
\end{array}\right] u+\left[\begin{array}{c}
0 \\
d_{\lambda_{P}} \\
d_{\alpha} \\
d_{\omega_{z}}
\end{array}\right], \\
\mathbf{y} & =\left[\begin{array}{l}
x_{3} \\
x_{4}
\end{array}\right],
\end{aligned}
$$

where $a_{22}=-2 \dot{R}_{P} / R_{P}, a_{23}=-\left(\rho V^{2} S c_{y}^{\alpha}+2 P\right) \cos \gamma / 2 m R_{P}$, $a_{33}=-\left(\rho V^{2} S c_{y}^{\alpha}+2 P\right) / 2 m V, a_{43}=\rho V^{2} \operatorname{Scm}_{z}^{\alpha} / 2 I_{z}$, and $b_{4}=$ $\rho V^{2} S \bar{c} m_{z}^{\delta_{e}} / 2 I_{z} \cdot d_{\lambda_{P}}, d_{\alpha}$, and $d_{\omega_{z}}$ are all unknown bounded variables.

To design the control law with adaptive SMC method, IGC model should be simplified into a standard type, and it can be presented as follows.

Consider $\overline{\mathbf{x}}=\left[x_{1 z}, x_{2 z}, x_{3 z}, x_{4 z}\right]^{T}$ and $u_{z}=\delta_{e}$, and we can obtain

$$
\begin{aligned}
\dot{\overline{\mathbf{x}}}=\left[\begin{array}{cccc}
0 & 1 & 0 & 0 \\
0 & 0 & 1 & 0 \\
0 & 0 & 0 & 1 \\
0 & a_{2 z} & a_{3 z} & a_{4 z}
\end{array}\right] \overline{\mathbf{x}}+\left[\begin{array}{c}
0 \\
0 \\
0 \\
b_{4 z}
\end{array}\right] u_{z}+\left[\begin{array}{c}
0 \\
d_{2} \\
d_{3} \\
d_{4}
\end{array}\right], \\
\mathbf{y}=\left[x_{1 z}, x_{2 z}\right]^{T},
\end{aligned}
$$

where

$$
\begin{aligned}
x_{1 z}= & \lambda_{P}-\lambda_{q p}, \\
\dot{x}_{1 z}= & x_{2 z}=\dot{\lambda}_{P}, \\
\dot{x}_{2 z}= & x_{3 z}+d_{2}, \\
\dot{x}_{3 z}= & x_{4 z}+d_{3}, \\
\dot{x}_{4 z}= & a_{2 z} x_{2 z}+a_{3 z} x_{3 z}+a_{4 z} x_{4 z}+b_{4 z} u_{z}+d_{4}, \\
a_{2 z}= & \ddot{a}_{22}-\dot{a}_{22} a_{33}-a_{22} \dot{a}_{33}-a_{22} a_{43}-\frac{2 \dot{a}_{22} \dot{a}_{23}}{a_{23}} \\
& -\frac{a_{22} \ddot{a}_{23}}{a_{23}}+\frac{2 a_{22} \dot{a}_{23}^{2}}{a_{23}^{2}}+\frac{a_{22} a_{33} \dot{a}_{23}}{a_{23}}, \\
a_{3 z}= & 2 \dot{a}_{22}+\dot{a}_{33}+a_{43}-a_{22} a_{33}+\frac{\ddot{a}_{23}}{a_{23}}-\frac{2 \dot{a}_{23}^{2}}{a_{23}^{2}} \\
& -\frac{2 a_{22} \dot{a}_{23}}{a_{23}}-\frac{a_{33} \dot{a}_{23}}{a_{23}}, \\
a_{4 z}= & a_{22}+a_{33}+\frac{2 \dot{a}_{23}}{a_{23}}, \\
b_{4 z}= & a_{23} b_{4}, \\
d_{4}= & \left(\dot{a}_{22}+a_{22}^{2}\right) d_{\lambda_{P}}+\left(\dot{a}_{23}+a_{22} a_{23}+a_{23} a_{33}\right) d_{\alpha} \\
& +a_{23} d_{\omega_{z}} .
\end{aligned}
$$

4.2.2. Roll Channel. According to the relative movement between UCAV and the target described by (2) we obtain

$$
\begin{aligned}
\ddot{\lambda}_{L}= & -\frac{2 \dot{R}_{L}}{R_{L}} \dot{\lambda}_{L}+\frac{\cos \left(\theta_{T L}-\lambda_{L}\right)}{R_{L}} a_{T L} \\
& -\frac{\cos \left(\theta_{U L}-\lambda_{L}\right)}{R_{L}} a_{U L} .
\end{aligned}
$$

In the terminal phase of attack occupation, $\lambda_{L} \approx \theta_{U L}$. Considering the target's acceleration as an unknown bounded variable, we obtain

$$
\ddot{\lambda}_{L}=-\frac{2 \dot{R}_{L}}{R_{L}} \dot{\lambda}_{L}-\frac{a_{U L}^{\prime}}{R_{L}}+\frac{d_{\lambda_{L 1}}}{R_{L}}
$$

where $a_{U L}^{\prime}=\cos \left(\theta_{U L}-\lambda_{L}\right) a_{U L} \approx a_{U L}$. Furthermore, according to the dynamic analysis of the UCAV, its roll angle is always restricted in small scope in the process of autonomous attack occupation. Hence, let $\sin \gamma \approx \gamma$ and $a_{U L}^{\prime}$ can be attained as

$$
a_{U L}^{\prime} \approx-\frac{\left(\rho V_{U L}^{2} S c_{y}^{\alpha}+2 P\right) \alpha \gamma}{2 m}+d_{a_{U L}}
$$

In (23) $d_{a_{U L}}$ is the approximate error of lateral acceleration in the lateral plane. 
Substituting (23) into (22) yields

$$
\begin{aligned}
\ddot{\lambda}_{L} & =-\frac{2 \dot{R}_{L}}{R_{L}} \dot{\lambda}_{L}+\frac{\left(\rho V_{U L}^{2} S c_{y}^{\alpha}+2 P\right) \alpha \gamma}{2 m R_{L}}+\frac{d_{\lambda_{L 1}}-d_{a_{U L}}}{R_{L}} \\
& =-\frac{2 \dot{R}_{L}}{R_{L}} \dot{\lambda}_{L}+\frac{\left(\rho V_{U L}^{2} S c_{y}^{\alpha}+2 P\right) \alpha \gamma}{2 m R_{L}}+d_{\lambda_{L}},
\end{aligned}
$$

where $d_{\lambda_{L}}$ denotes an unknown bounded variable, which contains the approximate error of lateral acceleration and the unknown information of target's maneuver.

According to the gesture constraints of UCAV in lateral plane, which have been analyzed in Section 4.1, we can obtain

$$
\lambda_{q l}=\theta_{q l}-\frac{\pi}{8}
$$

Define the state variables, control variable, and output variables as follows:

$$
\begin{aligned}
& \mathbf{x}=\left[x_{1}, x_{2}, x_{3}, x_{4}\right]^{T}=\left[\lambda_{L}-\lambda_{q l}, \dot{\lambda}_{L}, \gamma, \omega_{x}\right]^{T}, \\
& u=\delta_{a}, \\
& \mathbf{y}=\left[x_{3}, x_{4}\right]^{T}=\left[\gamma, \omega_{x}\right]^{T} .
\end{aligned}
$$

Combining (26) with (24) and considering the coupling between the roll channel and other channels as unknown bounded variable, we can obtain the IGC model of roll channel as follows:

$$
\begin{aligned}
{\left[\begin{array}{c}
\dot{x}_{1} \\
\dot{x}_{2} \\
\dot{x}_{3} \\
\dot{x}_{4}
\end{array}\right]=\left[\begin{array}{cccc}
0 & 1 & 0 & 0 \\
0 & a_{22} & a_{23} & 0 \\
0 & 0 & 0 & 1 \\
0 & 0 & 0 & 0
\end{array}\right]\left[\begin{array}{l}
x_{1} \\
x_{2} \\
x_{3} \\
x_{4}
\end{array}\right]+\left[\begin{array}{l}
0 \\
0 \\
0 \\
b_{4}
\end{array}\right] u+\left[\begin{array}{c}
0 \\
d_{\lambda_{L}} \\
d_{\gamma} \\
d_{\omega_{x}}
\end{array}\right], } \\
\mathbf{y}=\left[\begin{array}{l}
x_{3} \\
x_{4}
\end{array}\right],
\end{aligned}
$$

where $a_{22}=-2 \dot{R}_{L} / R_{L}, a_{23}=\left(\rho V_{U L}^{2} S c_{y}^{\alpha}+2 P\right) \alpha / 2 m R_{L}$, and $b_{4}=$ $\rho V^{2} \operatorname{Sbm}_{x}^{\delta_{a}} / 2 I_{x} . d_{\lambda_{L}}, d_{\gamma}$, and $d_{\omega_{x}}$ are all unknown bounded variables.

The IGC model of roll channel has the same form as that of the pitch channel, and its standard form can be got by using the same method in the analysis of pitch channel.

4.2.3. Yaw Channel. As to yaw channel, define the state variables, control variable, and output variables as follows

$$
\begin{aligned}
& \mathbf{x}=\left[x_{1}, x_{2}\right]^{T}=\left[\beta, \omega_{y}\right]^{T}, \\
& u=\delta_{r}, \\
& \mathbf{y}=\left[x_{1}, x_{2}\right]^{T}=\left[\beta, \omega_{y}\right]^{T} .
\end{aligned}
$$

Seeing the coupling between the yaw channel and other channels as unknown bounded variable, we can obtain

$$
\begin{aligned}
{\left[\begin{array}{l}
\dot{x}_{1} \\
\dot{x}_{2}
\end{array}\right] } & =\left[\begin{array}{ll}
a_{11} & 1 \\
a_{21} & 0
\end{array}\right]\left[\begin{array}{l}
x_{1} \\
x_{2}
\end{array}\right]+\left[\begin{array}{l}
0 \\
b_{2}
\end{array}\right] u+\left[\begin{array}{l}
d_{\beta} \\
d_{\omega_{y}}
\end{array}\right], \\
\mathbf{y} & =\left[\begin{array}{l}
x_{1} \\
x_{2}
\end{array}\right],
\end{aligned}
$$

where $a_{11}=\left(\rho V^{2} S c_{z}^{\beta}-2 P\right) / 2 m V, a_{21}=\rho V^{2} S b m_{y}^{\beta} / 2 I_{y}$, and $b_{2}=\rho V^{2} \operatorname{Sbm}_{y}^{\delta_{r}} / 2 I_{y}$.

In order to standardize the control system, we further set the state variables $\overline{\mathbf{x}}=\left[x_{1 y}, x_{2 y}\right]^{T}$ and control variable $u_{y}=$ $\delta_{r}$. Then, the state representation can be described as follows:

$$
\begin{aligned}
& \dot{\overline{\mathbf{x}}}=\left[\begin{array}{cc}
0 & 1 \\
a_{1 y} & a_{2 y}
\end{array}\right] \overline{\mathbf{x}}+\left[\begin{array}{c}
0 \\
b_{2 y}
\end{array}\right] u_{y}+\left[\begin{array}{l}
d_{\beta} \\
\dot{d}_{\beta}
\end{array}\right], \\
& \mathbf{y}=\left[x_{1 y}, x_{2 y}\right]^{T},
\end{aligned}
$$

where

$$
\begin{aligned}
& x_{1 y}=\beta, \\
& x_{2 y}=\dot{\beta}, \\
& a_{1 y}=a_{21}, \\
& a_{2 y}=a_{11} .
\end{aligned}
$$

4.3. A SMC Law for the IGC System of UCAV. By taking external disturbances and internal parameter perturbation into consideration, a sliding mode control law is designed to make the IGC system of UCAV fleetly reach the sliding surface with a stable sliding mode. As to the process of autonomous attack occupation, the purpose of the SMC law is to make the LOS angular rate between UCAV and the optimum attack position approach 0 in the condition that the UCAV meets the gesture constraints at the attack moment.

$\mathrm{SMC}$ is an effective robust control algorithm since it is insensitive to model uncertainties, external disturbances, and parameter variations [27]. In SMC, the state of the control system is first guided to a designed surface (i.e., the sliding mode surface) and then it is forced there with a shifting law [28]. The control strategy of SMC is determined by the sum of two control laws: switching and equivalent control laws. The switching control law is discontinuous, and it enforces the system state to the predetermined sliding mode surface in a finite time. When the sliding mode occurs, the closedloop system becomes insensitive to parameter variations and robust to matched disturbances by means of the equivalent control law which is usually linear [29].

4.3.1. Design of the SMC Law for IGC System in Pitch and Roll Channels. As to the IGC system denoted by (19), set 
$e_{z}=x_{1 z}=\lambda_{P}-\lambda_{p q}$ and $u_{z}=\delta_{e}$ and the sliding surface can be designed as

$$
\begin{aligned}
S_{z} & =\left(\frac{d}{d t}+\varepsilon_{z}\right)^{3} e_{z}=\sum_{l=0}^{3} C_{3}^{l} \varepsilon_{z}^{l} e_{z}^{(3-l)} \\
& =\varepsilon_{z}^{3} e_{z}+3 \varepsilon_{z}^{2} \dot{e}_{z}+3 \varepsilon_{z} \ddot{e}_{z}+\ddot{e}_{z},
\end{aligned}
$$

where $\varepsilon_{z}$ is a positive constant called sliding mode coefficient and $d / d t$ is the differential operator with respect to $e_{z}$. Then, the control law can be designed as follows:

$$
u_{z}=\frac{1}{b_{4 z}}(\widehat{u}+\widetilde{u}),
$$

where

$$
\begin{aligned}
& \widehat{u}=-\left(a_{2 z} x_{2 z}+a_{3 z} x_{3 z}+a_{4 z} x_{4 z}\right)-\sum_{l=1}^{3} C_{3}^{l} \varepsilon_{z}^{l} e_{z}^{(4-l)}, \\
& \widetilde{u}=-k_{z 1} \frac{\left|\dot{R}_{P}\right|}{R_{P}} S_{z}-k_{z 2} \operatorname{sgn}\left(S_{z}\right), \quad k_{z 2}=\widetilde{d}_{z}+\varsigma_{z} .
\end{aligned}
$$

In (34), $\varsigma_{z}$ is a designed positive constant called controller coefficient and $\widetilde{d}_{z} \geq\left|3 \varepsilon_{z}^{2} d_{2}+3 \varepsilon_{z} d_{3}+d_{4}\right|$ is the bound of unknown uncertainties.

Furthermore, the existence, accessibility, and stability of the sliding mode are analyzed as follows.

Consider the following Lyapunov function candidate:

$$
V=\frac{1}{2} S_{z}^{2}
$$

By differentiating (32) with respect to time, it implies

$$
\begin{aligned}
\dot{S}_{z}= & \sum_{l=0}^{3} C_{3}^{l} \varepsilon_{z}^{l} \dot{e}_{z}^{(3-l)}=\sum_{l=1}^{3} C_{3}^{l} \varepsilon_{z}^{l} e_{z}^{(4-l)}+e_{z} \\
= & \sum_{l=1}^{3} C_{3}^{l} \varepsilon_{z}^{l} e_{z}^{(4-l)}+a_{2 z} x_{2 z}+a_{3 z} x_{3 z}+a_{4 z} x_{4 z}+b_{4 z} u_{z} \\
& +3 \varepsilon_{z}^{2} d_{2}+3 \varepsilon_{z} d_{3}+d_{4} \\
= & -k_{z 1} \frac{\left|\dot{R}_{P}\right|}{R_{P}} S_{z}-k_{z 2} \operatorname{sgn}\left(S_{z}\right)+3 \varepsilon_{z}^{2} d_{2}+3 \varepsilon_{z} d_{3}+d_{4} .
\end{aligned}
$$

Substitute (36) into (35), and we obtain

$$
\begin{aligned}
\dot{V}= & S_{z} \dot{S}_{z} \\
= & -k_{z 1} \frac{\left|\dot{R}_{P}\right|}{R_{P}} S_{z}^{2}-k_{z 2}\left|S_{z}\right|+\left(3 \varepsilon_{z}^{2} d_{2}+3 \varepsilon_{z} d_{3}+d_{4}\right) S_{z} \\
= & -k_{z 1} \frac{\left|\dot{R}_{P}\right|}{R_{P}} S_{z}^{2}-\left(\tilde{d}_{z}+\varsigma_{z}\right)\left|S_{z}\right| \\
& +\left(3 \varepsilon_{z}^{2} d_{2}+3 \varepsilon_{z} d_{3}+d_{4}\right) S_{z} \\
\leq & -k_{z 1} \frac{\left|\dot{R}_{P}\right|}{R_{P}} S_{z}^{2}-\varsigma_{z}\left|S_{z}\right| \leq-\varsigma_{z}\left|S_{z}\right| .
\end{aligned}
$$

Therefore, the system is stable and it can reach the sliding surface in finite time.

The design of control law for roll channel is similar to that of pitch channel in format. However, its specific parameters are decided by the IGC model of roll channel.

4.3.2. Design of the SMC Law for IGC Model in Yaw Channel. As to the IGC system denoted by (30), set $e_{y}=x_{1 y}=\beta$ and $u_{y}=\delta_{r}$ and the sliding surface can be designed as follows:

$$
S_{y}=\left(\frac{d}{d t}+\varepsilon_{y}\right) e_{y}=\varepsilon_{y} e_{y}+\dot{e}_{y}
$$

where $\varepsilon_{y}$ is a positive constant called sliding mode coefficient and $d / d t$ is the differential operator with respect to $\varepsilon_{y}$. Then, the control law yields

$$
u_{y}=\frac{1}{b_{2 y}}(\widehat{u}+\widetilde{u}) \text {, }
$$

where

$$
\begin{aligned}
& \widehat{u}=-a_{1 y} e_{y}-\left(a_{2 y}+\varepsilon_{y}\right) \dot{e}_{y}, \\
& \widetilde{u}=-k_{y 1} S_{y}-k_{y 2} \operatorname{sgn}\left(S_{y}\right), \quad k_{y 2}=\widetilde{d}_{y}+\varsigma_{y} .
\end{aligned}
$$

In (40), $\varsigma_{y}$ is a designed positive constant which is called controller coefficient and $\tilde{d}_{y} \geq\left|\varepsilon_{y} d_{\beta}+\dot{d}_{\beta}\right|$ is the bound of unknown uncertainties.

Consider the following Lyapunov function candidate:

$$
V=\frac{1}{2} S_{y}^{2}
$$

By differentiating (38) with respect to time, it implies

$$
\begin{aligned}
\dot{S}_{y} & =\varepsilon_{y} \dot{e}_{y}+\ddot{e}_{y} \\
& =\varepsilon_{y} \dot{e}_{y}+a_{1 y} e_{y}+a_{2 y} \dot{e}_{y}+b_{2 y} u_{y}+\varepsilon_{y} d_{\beta}+\dot{d}_{\beta} \\
& =-k_{y 1} S_{y}-k_{y 2} \operatorname{sgn}\left(S_{y}\right)+\varepsilon_{y} d_{\beta}+\dot{d}_{\beta} .
\end{aligned}
$$

Substitute (42) into (41), and we obtain

$$
\begin{aligned}
\dot{V} & =S_{y} \dot{S}_{y}=-k_{y 1} S_{y}^{2}-k_{y}\left|S_{y}\right|+\left(\varepsilon_{y} d_{\beta}+\dot{d}_{\beta}\right) S_{y} \\
& =-k_{y 1} S_{y}^{2}-\left(\tilde{d}_{y}+\varsigma_{y}\right)\left|S_{y}\right|+\left(\varepsilon_{y} d_{\beta}+\dot{d}_{\beta}\right) S_{y} \\
& \leq-k_{y 1} S_{y}^{2}-\varsigma_{y}\left|S_{y}\right| \leq-\varsigma_{y}\left|S_{y}\right| .
\end{aligned}
$$

Therefore, the system is stable and it can reach the sliding surface in finite time.

In order to further smooth the control process, we replace the switching function $\operatorname{sgn}(S)$ with a saturation function $\operatorname{sat}(S / \phi)$, in which $\phi>0$ denotes the width of boundary 


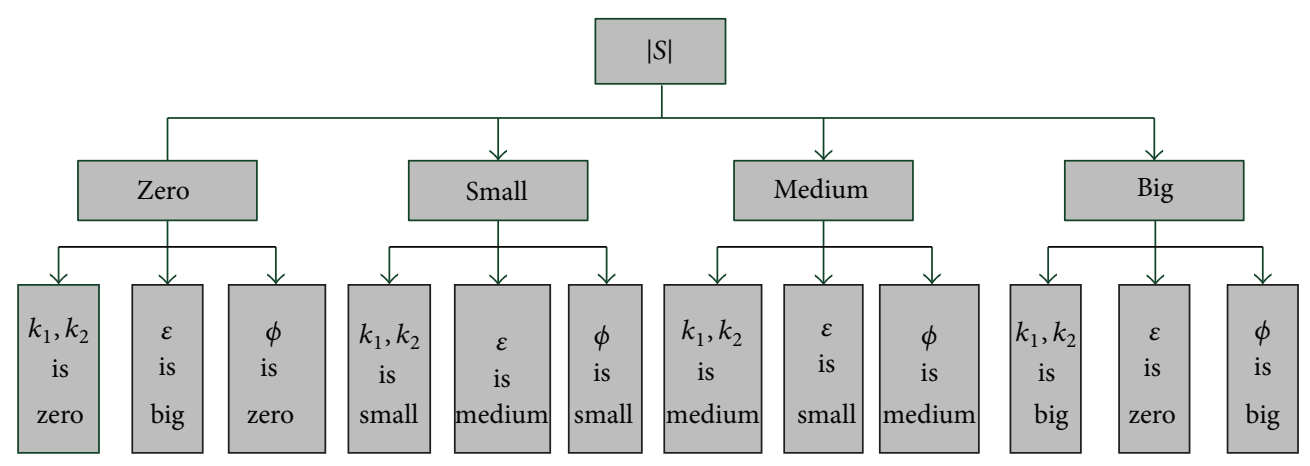

FIGURE 11: Regulating law of main parameters in the designed control system.

layer. Then the designed control laws of pitch, yaw, and roll channels are shown as follows:

$$
\begin{aligned}
\delta_{e} & =\frac{1}{b_{4 z}}\left[-\left(a_{2 z} x_{2 z}+a_{3 z} x_{3 z}+a_{4 z} x_{4 z}\right)\right. \\
& \left.-\sum_{l=1}^{3} C_{3}^{l} \varepsilon_{z}^{l} e_{z}^{(4-l)}-k_{z 1} \frac{\left|\dot{R}_{P}\right|}{R_{P}} S_{z}-k_{z 2} \operatorname{sat}\left(\frac{S_{z}}{\phi_{z}}\right)\right], \\
\delta_{r} & =\frac{1}{b_{2}}\left[-\varepsilon_{y} \dot{e}_{y}-k_{y 1} S_{y}-k_{y 2} \operatorname{sat}\left(\frac{S_{y}}{\phi_{y}}\right)\right], \\
\delta_{a} & =\frac{1}{b_{4 x}}\left[-\left(a_{2 x} x_{2 x}+a_{3 x} x_{3 x}+a_{4 x} x_{4 x}\right)\right. \\
& \left.-\sum_{l=1}^{3} C_{3}^{l} \varepsilon_{x}^{l} e_{x}^{(4-l)}-k_{x 1} \frac{\left|\dot{R}_{L}\right|}{R_{L}} S_{x}-k_{x 2} \mathrm{sat}\left(\frac{S_{x}}{\phi_{x}}\right)\right],
\end{aligned}
$$

where $\delta_{e}, \delta_{r}$, and $\delta_{a}$ are the angle of elevator, rudder, and aileron of the UCAV, respectively.

\subsection{Regulation of Parameters in the Designed Control System of} $U C A V$. Taking a close look into the designed control system, we find that the sliding mode, response time, and control error of it are mainly affected by the system parameters $k_{1}$, $k_{2}$, $\varepsilon$, and $\phi$. (1) Larger values of $k_{1}$ and $k_{2}$ shorten the time that control system spends to reach the sliding mode surface. However, they bring obvious chattering phenomenon and more consumption of control energy. So we prefer larger values of $k_{1}$ and $k_{2}$ when the system state is far from sliding mode surface and smaller values of $k_{1}$ and $k_{2}$ are more suitable on the contrary. (2) After reaching the sliding mode state, the control system tends to be stable in negative exponential form with parameter $\varepsilon$, and we prefer a larger value of $\varepsilon$ to save the convergence time. But a large value of $\varepsilon$ may trigger the unmodeled high frequency state when the control system is not in the sliding mode state, and a smaller $\varepsilon$ is preferred in this case. (3) The width of boundary layer $\phi$ can indirectly handle the steady-state error of our control system. A smaller $\phi$ brings smaller steady-state error but higher frequency of switching control and more consumption of control energy. Therefore, we consider a larger $\phi$ when the system state is far from sliding mode surface to speed up the reaching process. On the contrary, a smaller $\phi$ is preferred to reduce the steady-state error when our control system is reaching the sliding mode state.

Moreover, we find that all of the parameters have a close relationship with the sliding surface $S$. Therefore, we design a law in which main parameters of the designed control system are regulated adaptively with the change of sliding surface $S$ to meet better control performance [30]. Figure 11 shows the regulating law of the parameters in the designed control system.

In this paper, a negative exponential function is used, within which $|S|$ is an independent variable. The variable $|S|$ can adaptively regulate the main parameters in the designed control system. According to the regulating law shown in Figure 11, the detailed description of $k_{1}, k_{2}, \varepsilon$, and $\phi$ is as follows:

$$
\begin{aligned}
\varepsilon & =\varepsilon_{\max } e^{-S^{2} / 2}, \\
k_{1} & =k_{1 \max }+\left(k_{1 \min }-k_{1 \max }\right) e^{-S^{2} / 2}, \\
k_{2} & =k_{2 \max }+\left(k_{2 \min }-k_{2 \max }\right) e^{-S^{2} / 2}, \\
\phi & =\phi_{\max }+\left(\phi_{\min }-\phi_{\max }\right) e^{-S^{2} / 2} .
\end{aligned}
$$

Considering the regulating law described above, the control input in the pitch channel is $u_{z}=\left(1 / b_{4 z}\right)(\widehat{u}+\widetilde{u})$, where the linear control input part $\widehat{u}$ and the discontinuous control input part $\tilde{u}$ are as follows:

$$
\begin{aligned}
\widehat{u}= & -\left(a_{2 z} x_{2 z}+a_{3 z} x_{3 z}+a_{4 z} x_{4 z}\right)-\sum_{l=1}^{3} C_{3}^{l} \varepsilon_{z}^{l} e_{z}^{(4-l),} \\
\widetilde{u}= & \operatorname{sgn}\left(1+\sum_{l=1}^{3} l C_{3}^{l} \varepsilon_{z}^{l} e_{z}^{(3-l)} S_{z}\right) \\
& \cdot\left(-k_{z 1} \frac{\left|\dot{R}_{P}\right|}{R_{P}} S_{z}-k_{z 2} \operatorname{sgn}\left(S_{z}\right)\right),
\end{aligned}
$$

where $\varepsilon_{z}, k_{z 1}$, and $k_{z 2}$ are in the format described in (45). In this case, we further set $k_{z 2 \min } \geq \widetilde{d}_{z}+\varsigma_{z}$, where $\varsigma_{z}$ is the controller coefficient and $\tilde{d}_{z} \geq\left|3 \varepsilon_{z}^{2} d_{2}+3 \varepsilon_{z} d_{3}+d_{4}\right|$ is the 
bound of unknown uncertainties as analyzed before. Then, by differentiating (32) with respect to time, it implies

$$
\dot{S}_{z}=\sum_{l=0}^{3} C_{3}^{l} \varepsilon_{z}^{l} \dot{e}_{z}^{(3-l)}+\dot{\varepsilon}_{z} \sum_{l=1}^{3} l C_{3}^{l} \varepsilon_{z}^{l-1} e_{z}^{(3-l)}
$$

In addition, from the regulating law, $\dot{\varepsilon}_{z}$ can be presented as

$$
\dot{\varepsilon}_{z}=-\varepsilon_{z \max } e^{-S_{z}^{2} / 2} S_{z} \dot{S}_{z}=-\varepsilon_{z} S_{z} \dot{S}_{z}
$$

Substitute (46) and (48) into (47), and we obtain

$$
\dot{S}_{z}=\frac{\operatorname{sgn}\left(1+\sum_{l=1}^{3} l C_{3}^{l} \varepsilon_{z}^{l} e_{z}^{(3-l)} S_{z}\right)\left(-k_{z 1}\left(\left|\dot{R}_{P}\right| / R_{P}\right) S_{z}-k_{z 2} \operatorname{sgn}\left(S_{z}\right)\right)+3 \varepsilon_{z}^{2} d_{2}+3 \varepsilon_{z} d_{3}+d_{4}}{1+\sum_{l=1}^{3} l C_{3}^{l} \varepsilon_{z}^{l} e_{z}^{(3-l)} S_{z}}
$$

where $\varepsilon_{z}=\varepsilon_{z \max } e^{-S_{z}^{2} / 2}, k_{z 1}=k_{z 1 \max }+\left(k_{z 1 \min }-k_{z 1 \max }\right) e^{-S_{z}^{2} / 2}$, and $k_{z 2}=k_{z 2 \max }+\left(k_{z 2 \min }-k_{z 2 \max }\right) e^{-S_{z}^{2} / 2}$. Considering that $k_{z 2 \min } \geq \tilde{d}_{z}+\varsigma_{z}, \varsigma_{z}>0$ and $\widetilde{d}_{z} \geq\left|3 \varepsilon_{z}^{2} d_{2}+3 \varepsilon_{z} d_{3}+d_{4}\right|$, the derivative of Lyapunov function candidate can be denoted as follows:

$$
\begin{aligned}
\dot{V}= & S_{z} \dot{S}_{z}=\frac{\operatorname{sgn}\left(1+\sum_{l=1}^{3} l C_{3}^{l} \varepsilon_{z}^{l} e_{z}^{(3-l)} S_{z}\right)\left(-k_{z 1}\left(\left|\dot{R}_{P}\right| / R_{P}\right) S_{z}-k_{z 2} \operatorname{sgn}\left(S_{z}\right)\right)+3 \varepsilon_{z}^{2} d_{2}+3 \varepsilon_{z} d_{3}+d_{4}}{1+\sum_{l=1}^{3} l C_{3}^{l} \varepsilon_{z}^{l} e_{z}^{(3-l)} S_{z}} S_{z} \\
& \leq \frac{-\left(k_{z 1 \max }+\left(k_{z 1 \min }-k_{z 1 \max }\right) e^{-S_{z}^{2} / 2}\right)\left(\left|\dot{R}_{P}\right| / R_{P}\right) S_{z}^{2}-\left(\varsigma_{z}+k_{z 2 \max }\left(1-e_{z}^{-S_{z}^{2} / 2}\right)\right)\left|S_{z}\right|}{\left|1+\sum_{l=1}^{3} l C_{3}^{l} \varepsilon_{z}^{l} e_{z}^{(3-l)} S_{z}\right|} .
\end{aligned}
$$

Thus, the control law given by (46) and adaptive regulating law in (45) guarantee the reaching and sustaining of the sliding mode.

We further replace $\operatorname{sgn}\left(S_{z}\right)$ in (46) by the saturation function $\operatorname{sat}\left(S_{z} / \phi_{z}\right)$, where the width of boundary layer $\phi_{z}$ obeys the adaptive regulating law $\phi_{z}=\phi_{z \max }+\left(\phi_{z \min }-\right.$ $\left.\phi_{z \max }\right) e^{-S_{z}^{2} / 2}$. By regulating $\phi_{z}$ adaptively, the control system brings smaller steady-state error. At the same time, there is less chattering in the control process.

In the yaw channel and roll channel, we can draw the same conclusion as we discussed above.

\section{Simulation}

5.1. Simulation Scenario and Initial Conditions. Assume that the enemy aircraft implements " $\mathrm{S}$ " maneuver on a constant height $H_{T}=6 \mathrm{~km}$. Its velocity $V_{T}=300 \mathrm{~m} / \mathrm{s}$, and its lateral acceleration $a_{T}=10 \operatorname{sgn}\left(\sin \left(\omega_{T} t+\pi / 2\right)\right)$ within which $\omega_{T}=\pi / 5$. According to the conclusion of Section 3, the target position, namely, the optimum attack position that UCAV should maneuver to, is point $B$ or point $C$ in Figure 12. In addition, assume the UCAV's velocity $V_{U}=$ $300 \mathrm{~m} / \mathrm{s}$, and it approaches the enemy aircraft with negative height $\Delta H=-1 \mathrm{~km}$. The initial horizontal distance between them is $20 \mathrm{~km}$, and they are in the same vertical plane. The specific geometry relationship of them is illustrated in Figure 12.

The structure characteristics of the UCAV are shown in Table 1. The initial states of the UCAV and enemy aircraft are shown in Table 2 . Table 3 sets the key parameters of the designed control system. In addition, we set the amplitude of elevator, rudder, and aileron of the UCAV as $\pm 20^{\circ}$.

5.2. Simulation Results and Analysis. In this section, G\&C represents the traditional design that separates the guidance loop from the control loop, IGC represents the design of integrated guidance and control, and AIGC represents the IGC design with our proposed adaptive SMC method, which regulates key parameters of the IGC system timely. The simulation results are shown in Figures 13-24.

5.2.1. Pitch Channel. See Figures $13,14,15$, and 16.

\subsubsection{Yaw Channel. See Figures 17, 18, 19, and 20 .}

\subsubsection{Roll Channel. See Figures 21, 22, 23, and 24 .}

The simulation results of pitch channel show that, no matter what kind of designed control system is applied, when the UCAV arrives at the attack position, its attack angle and pitch rate converge to 0 . However, in the case of IGC and AIGC, the values of attack angle and pitch rate are relatively smaller in the whole process of attack occupation. What is more, they change more smoothly compared with that in the case of G\&C. Figure 15 further shows that the angle of UCAV's elevator is relatively small in the terminal phase of attack occupation in the case of IGC and AIGC. On the contrary, the angle of elevator is in saturated state for a long time in the case of G\&C. Because the response from autopilot lags behind the guidance command in the case of G\&C, therefore, from 


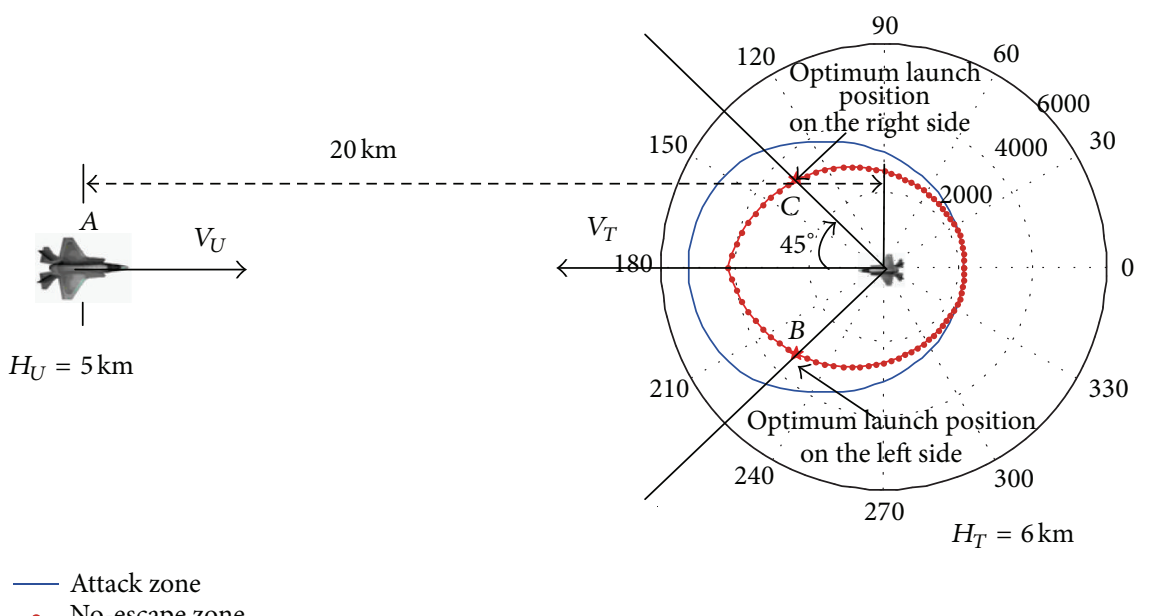

FIGURE 12: Initial scenario for simulation.

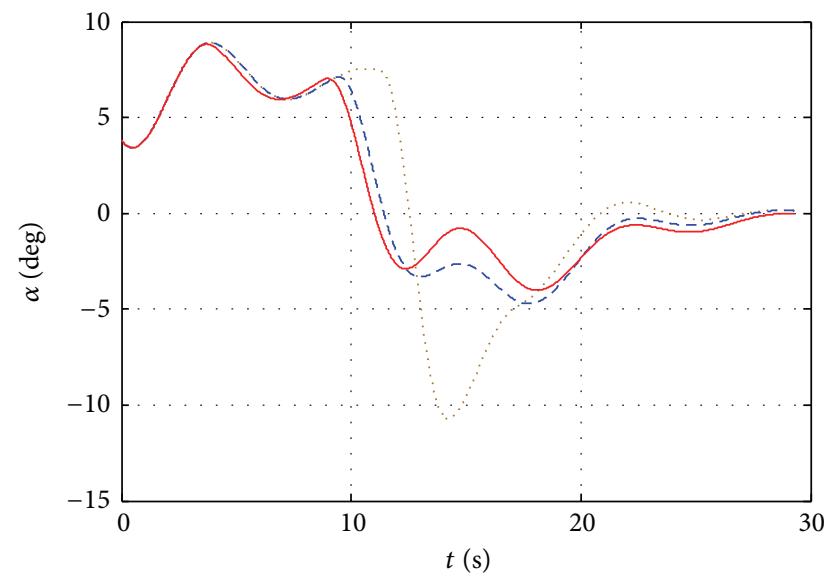

G\&C

- - IGC

- AIGC

FIgURe 13: Angle of attack.

TABLE 1: Structure characteristics of the UCAV.

\begin{tabular}{lcc}
\hline Intrinsic characteristics & Value & Unit \\
\hline Mass $(m)$ & 512 & $\mathrm{~kg}$ \\
\hline Moment of inertia & & \\
$\quad I_{x}$ & 1280 & $\mathrm{Kg} \cdot \mathrm{m}^{2}$ \\
$I_{y}$ & 8322 & $\mathrm{~kg} \cdot \mathrm{m}^{2}$ \\
$I_{z}$ & 7645 & $\mathrm{~kg} \cdot \mathrm{m}^{2}$ \\
$I_{x y}$ & 138 & $\mathrm{~kg} \cdot \mathrm{m}^{2}$ \\
\hline Wing span $(b)$ & 14.8 & $\mathrm{~m}$ \\
Wing area $(S)$ & 11.5 & $\mathrm{~m}$ \\
Mean aerodynamic chord $(\bar{c})$ & 0.777 & $\mathrm{~m}$ \\
\hline
\end{tabular}

the simulation results of pitch channel we can see that our proposed AIGC system can overcome the shortage of timelag in G\&C system, and it performs better than the simple IGC system.

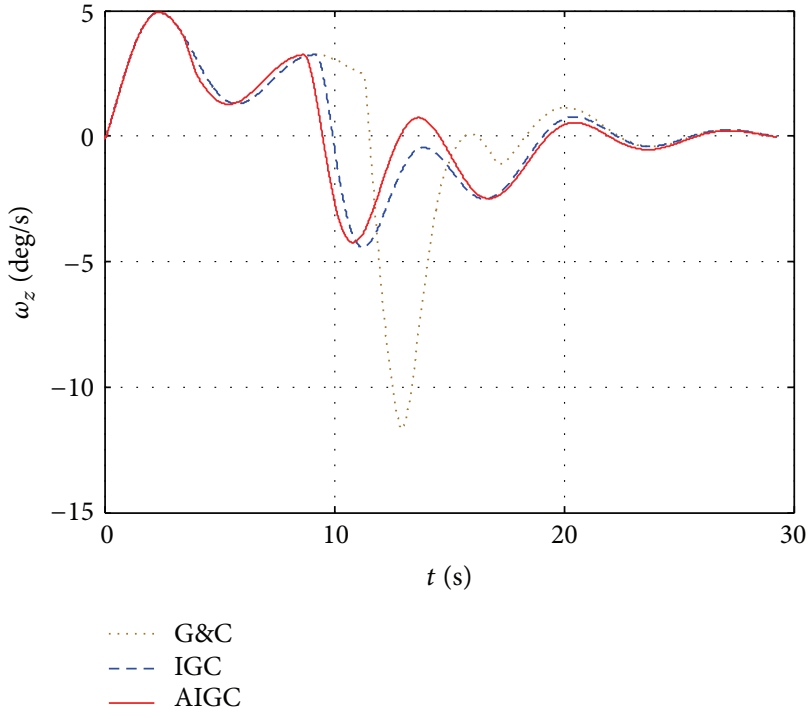

Figure 14: Pitch rate.

In the yaw channel, both the sideslip angle and yaw rate of UCAV change in a narrow range in the terminal phase of attack occupation. In addition, the range of sideslip angle is $\left[-1.5^{\circ}, 0.5^{\circ}\right]$, which further provides proof for the assumption that "lateral force caused by sideslip angle has little effect on the UCAV" in Section 4.2. Figure 19 further implies that during the process of autonomous attack occupation the angle of UCAV's rudder approaches 0 within 1 second, and it is always in the range of amplitude.

Figures 21 and 22 indicate that in the roll channel the roll angle and roll rate of UCAV change tempestuously during the process of autonomous attack occupation. This results from the constraints of UCAV's gesture at the attack moment and the limitation of aileron amplitude. On the other hand, both the roll angle and roll rate finally converge to 0 at the attack moment in the case of IGC and AIGC. However, in the case of G\&C, UCAV is not in a stable state at the attack moment and cannot fulfill the attack occupation task. 
TABLE 2: Initial state of UCAV and the enemy aircraft.

\begin{tabular}{|c|c|c|c|c|c|c|c|}
\hline Position $(\mathrm{km})$ & Value & $\begin{array}{l}\text { Velocity } \\
(\mathrm{m} / \mathrm{s})\end{array}$ & Value & $\begin{array}{l}\text { Acceleration } \\
\left(\mathrm{m} / \mathrm{s}^{2}\right)\end{array}$ & Value & $\begin{array}{c}\text { Angle (deg) and } \\
\text { angular velocity } \\
(\mathrm{deg} / \mathrm{s})\end{array}$ & Value \\
\hline$X_{U}$ & 0 & $V_{U x}$ & 300 & $a_{U x}$ & 0 & $\alpha$ & 4 \\
\hline$H_{U}$ & 5 & $V_{U y}$ & 0 & $a_{U y}$ & 0 & $\beta$ & 0 \\
\hline$Z_{U}$ & 0 & $V_{U z}$ & 0 & $a_{U z}$ & 0 & $\gamma$ & 0 \\
\hline$X_{T}$ & 20 & $V_{T x}$ & -300 & $a_{T x}$ & 0 & $\omega_{x}$ & 0 \\
\hline$H_{T}$ & 6 & $V_{T y}$ & 0 & $a_{T y}$ & 0 & $\omega_{y}$ & 0 \\
\hline$Z_{T}$ & 0 & $V_{T z}$ & 0 & $a_{T z}$ & 10 & $\omega_{z}$ & 0 \\
\hline
\end{tabular}

TABLE 3: Controller parameters of the UCAV.

\begin{tabular}{|c|c|c|c|c|c|c|c|c|c|c|c|}
\hline \multirow{2}{*}{ System parameters } & \multirow{2}{*}{$\varepsilon$} & \multirow{2}{*}{$k_{1}$} & \multirow{2}{*}{$k_{2}$} & \multirow{2}{*}{$\phi$} & \multicolumn{7}{|c|}{ Regulation law of system parameters } \\
\hline & & & & & $\varepsilon_{\max }$ & $k_{1 \text { max }}$ & $k_{1 \text { min }}$ & $k_{2 \max }$ & $k_{2 \min }$ & $\phi_{\max }$ & $\phi_{\min }$ \\
\hline Pitch channel & 1 & 1 & 2 & 0.2 & 1 & 10 & 1 & 20 & 2 & 20 & 0.2 \\
\hline Yaw channel & 0.5 & 0.2 & 0.4 & 0.1 & 0.5 & 10 & 0.2 & 20 & 0.4 & 20 & 0.1 \\
\hline Roll channel & 0.7 & 1 & 2 & 0.1 & 0.7 & 10 & 1 & 20 & 2 & 20 & 0.1 \\
\hline
\end{tabular}

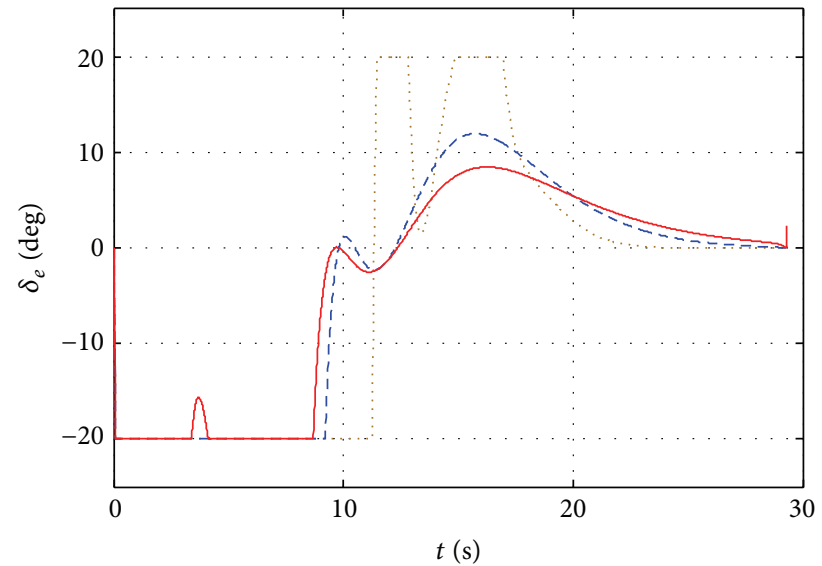

-.... G\&C

- - IGC

AIGC

Figure 15: Angle of elevator.

As the results in Figures 16, 20, and 24, in the case of AIGC, the sliding mode coefficient $\varepsilon$ converges to its maximum value. On the contrary, the reaching law coefficient $k_{1}$, control gain coefficient $k_{2}$, and width of boundary layer $\phi$ converge to their minimum value. These results are all in accordance with the regulation that we design for the key parameters of AIGC system. In addition, as shown in Figures 15,19 , and 23, in the case of AIGC the angle of elevator, rudder, and aileron of UCAV is relatively smaller than that when we apply G\&C or IGC. This is because the key parameters of AIGC system are time-varying during the autonomous attack occupation and the UCAV can maneuver according to the relative movement of enemy aircraft accurately and timely.

Let $U(t)=\left(\delta_{e}(t), \delta_{r}(t), \delta_{a}(t)\right)^{T}$, and we can obtain the equivalent consumption of control energy:

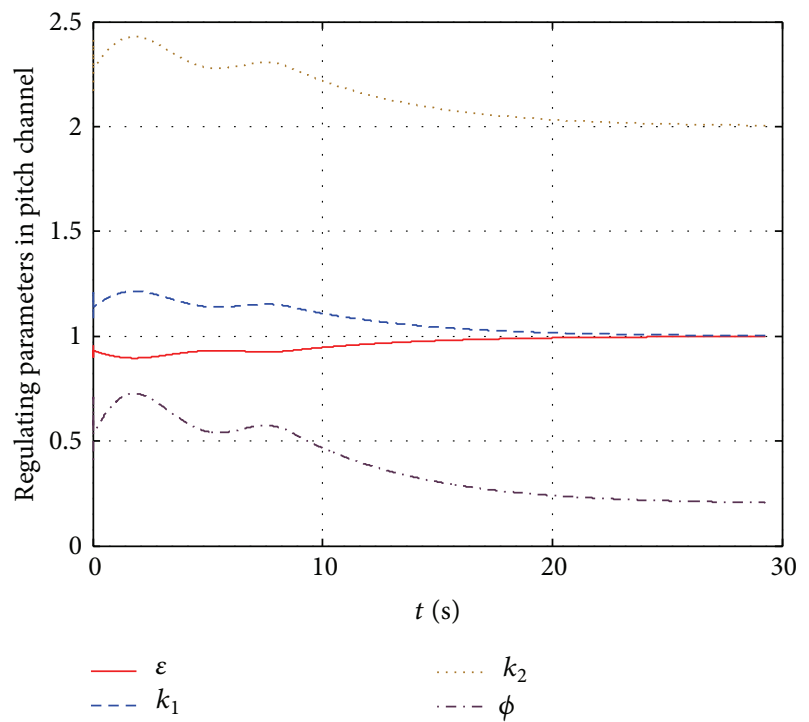

FIGURE 16: Key parameters in pitch channel.

$$
J=\int_{t_{0}}^{t_{f}} U^{T}(t) U(t) d t
$$

$\alpha_{f}, \beta_{f}, \gamma_{f}, \omega_{z f}, \omega_{y f}$, and $\omega_{x f}$, respectively, denote attack angle, sideslip angle, roll angle, pitch rate, yaw rate, and roll rate of the UCAV at the moment that it arrives at the optimum attack position. $|\alpha|_{\max },|\beta|_{\max },|\gamma|_{\max },\left|\omega_{z}\right|_{\max },\left|\omega_{y}\right|_{\max }$, and $\left|\omega_{x}\right|_{\text {max }}$, respectively, denote the maximum absolute value of UCAV's attack angle, sideslip angle, roll angle, pitch rate, yaw rate, and roll rate during the process of autonomous attack occupation.

According to the simulation results, the parameters of UCAV's performance in the case of three designed control systems are compared in Table 4. 


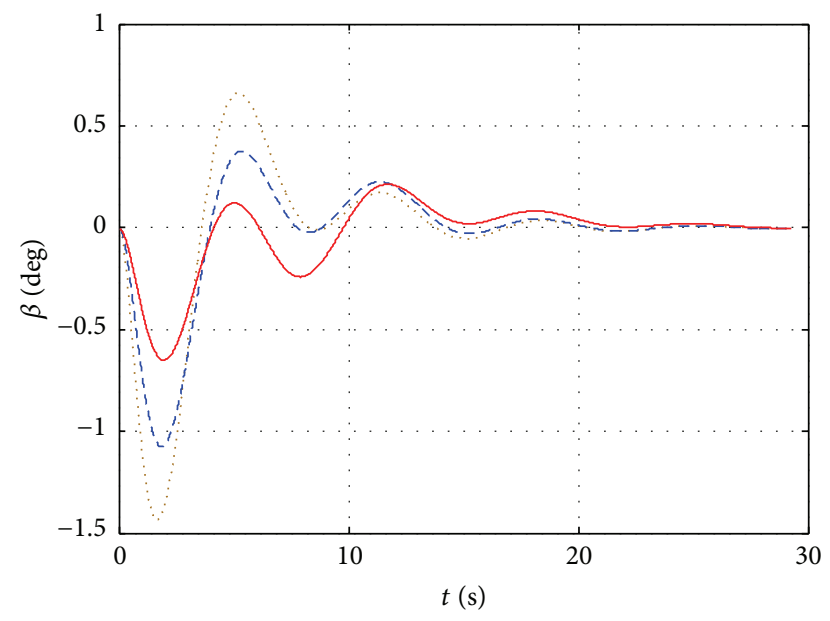

… G\&C

- - - IGC

- AIGC

FIGURE 17: Sideslip angle.

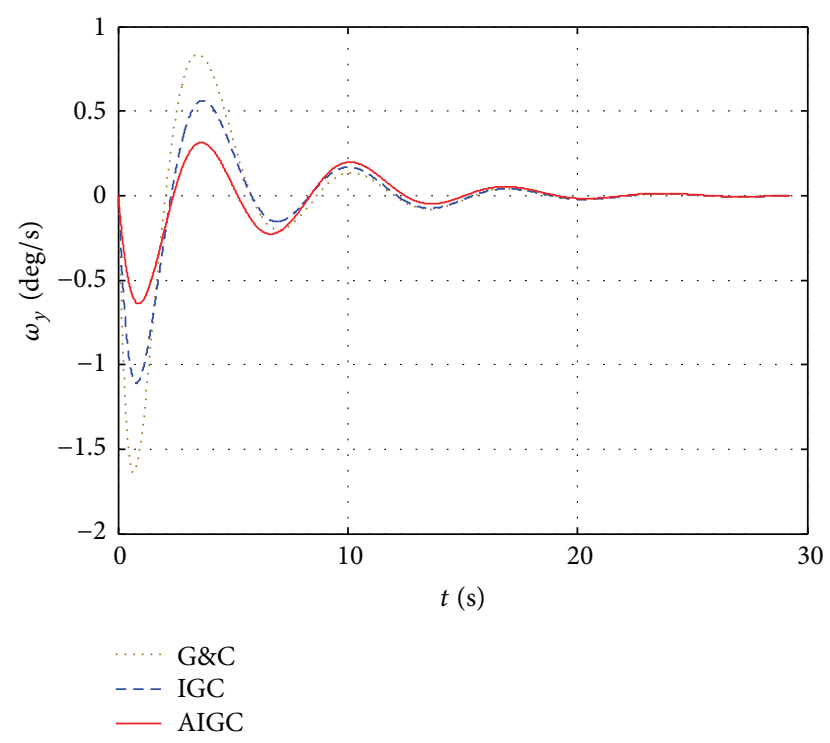

Figure 18: Yaw rate.

In Table 4, compared with G\&C and IGC, AIGC system can lead UCAV to the optimum attack position more stably and drive it to meet the gesture constraints more accurately. Namely, the kinematic parameters of UCAV are in a relatively smaller range in the process of autonomous attack occupation, and the attack angle, sideslip angle, roll angle, pitch rate, yaw rate, and roll rate of UCAV converge to 0 rapidly and accurately. What is more, AIGC system can economize control energy and weaken the chattering in the process of autonomous attack occupation. In our simulation, it economizes nearly $25 \%$ of the equivalent consumption of control energy $J$ compared with the G\&C and IGC system.

In the case of AIGC, the trajectory of autonomous attack occupation in three-dimensional space is shown in Figure 25.

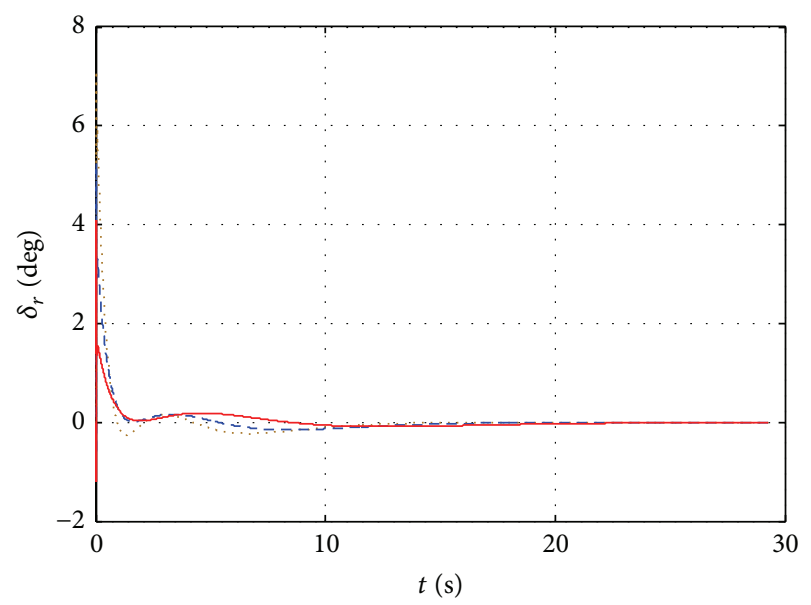

FIgURE 19: Angle of rudder.

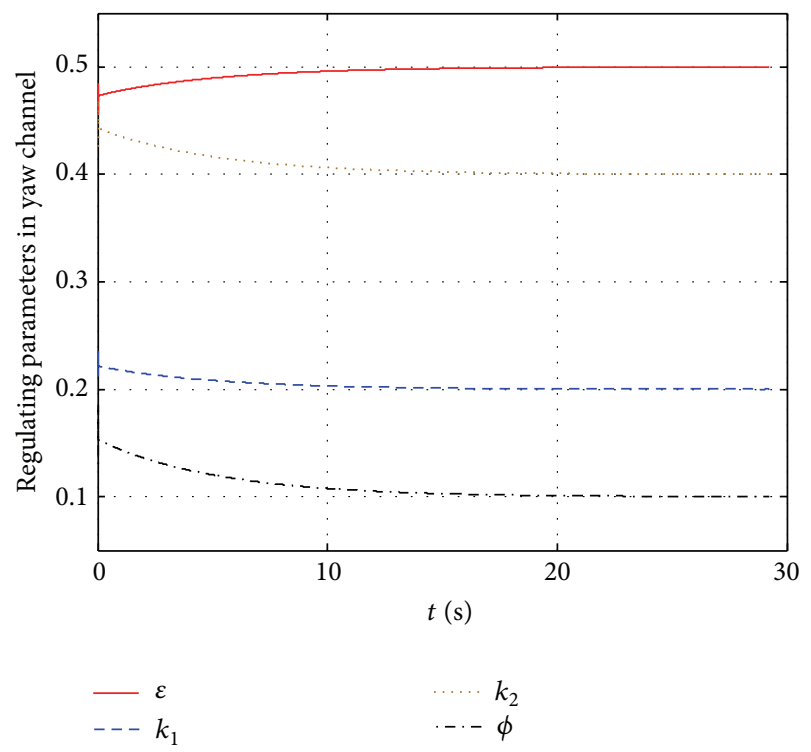

FIGURE 20: Key parameters in yaw channel.

\section{Conclusions}

In this paper, we have presented an approach for air-to-air autonomous attack occupation of UCAV, which calculates the optimum attack position and guides UCAV to it accurately with gesture constraints. The two main conclusions of this work are the following: (1) considering the requirement of precision, attack zone and no-escape zone can be calculated based on pattern search algorithm, and the optimum attack position can be designed by combining the calculated noescape zone with the practicality of air combat; (2) a novel adaptive SMC method is used to design the IGC system of UCAV, which can guide the UCAV to the optimum attack position accurately with gesture constraints at the attack moment. In the simulation, we demonstrate that compared with traditional methods the proposed approach can drive 


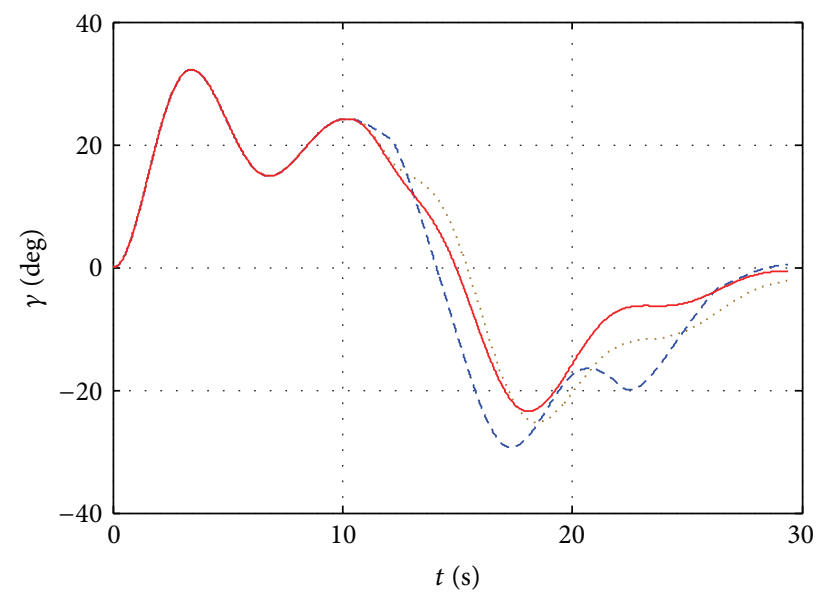

G\&C

- - IGC

- AIGC

FIGURE 21: Roll angle.

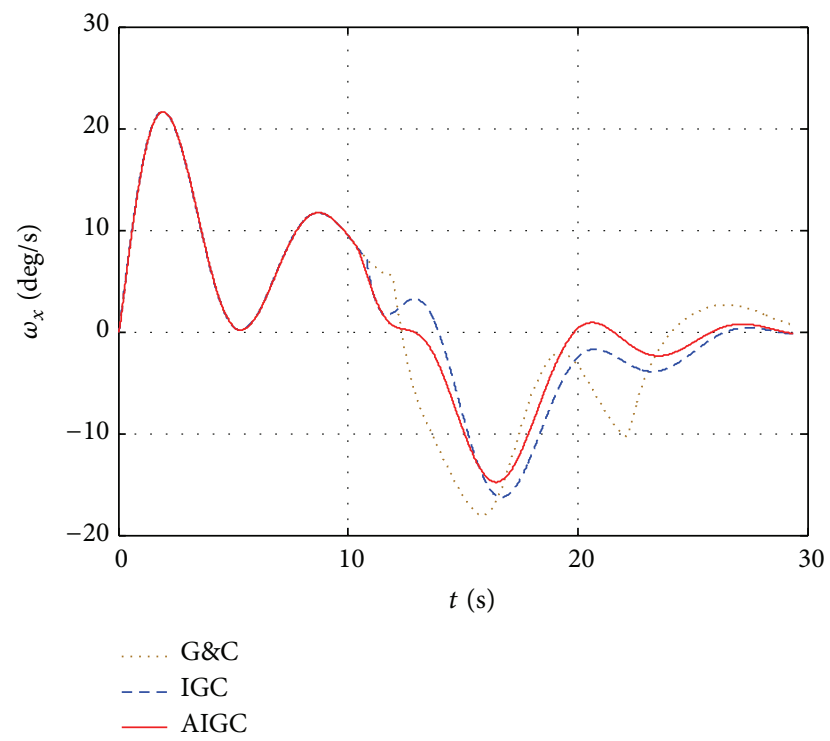

FIGURE 22: Roll rate.

the UCAV to fulfill the task of air-to-air autonomous attack occupation effectively with less consumption of control energy.

We believe that the approach of air-to-air autonomous attack occupation for UCAV in this work can inspire more new control systems of UCAV for the task of autonomous attack. For future work, we can explore not only suitable control systems for single UCAV in the task of autonomous attack but also new control and decision systems for multiUCAVs.

\section{Competing Interests}

The authors declare that there is no conflict of interests regarding the publication of this paper.

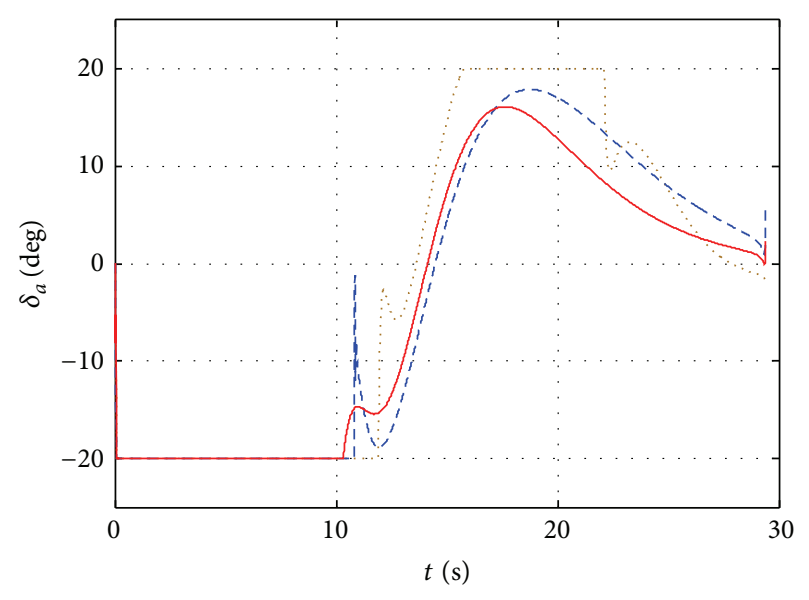

... G\&C

- - IGC

AIGC

FIgURE 23: Angle of aileron.

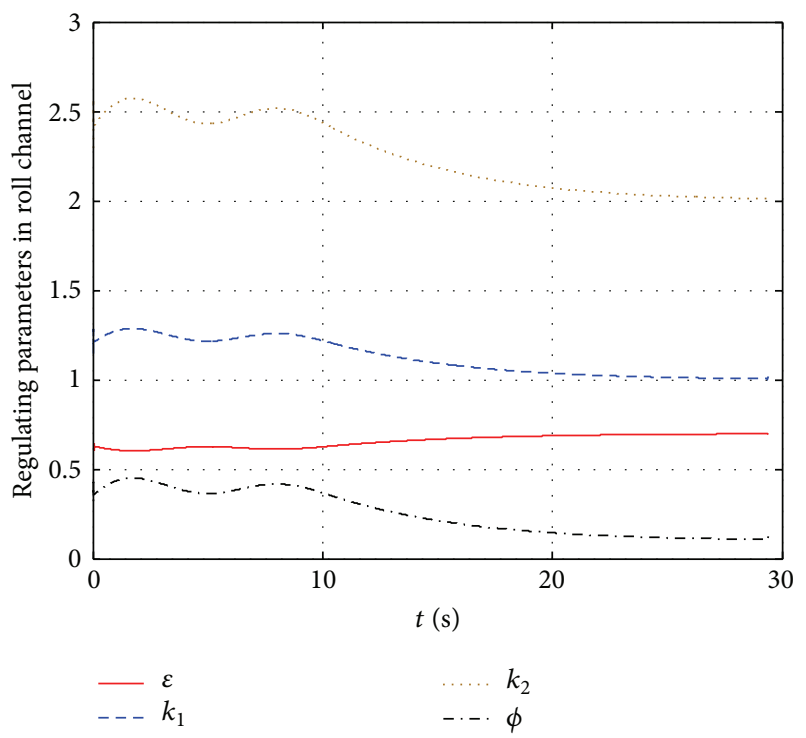

FIGURE 24: Key parameters in roll channel.

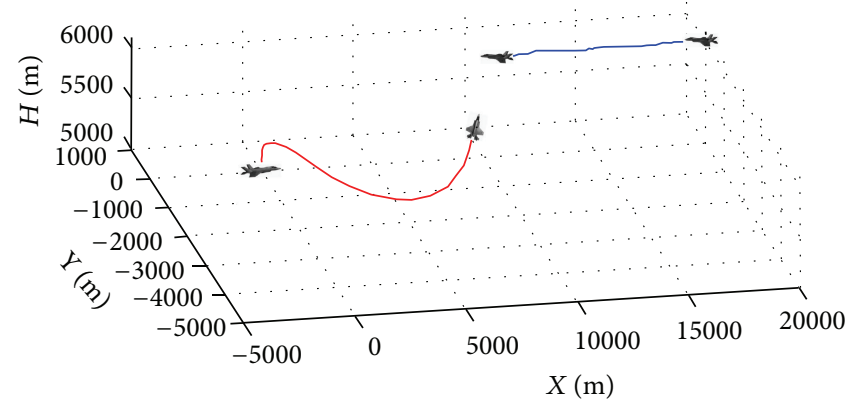

FIGURE 25: Trajectory of autonomous attack occupation in threedimensional space in the case of AIGC. 
TABLE 4: Parameters of UCAV's performance.

\begin{tabular}{lccc}
\hline Parameters & G\&C & IGC & AIGC \\
\hline$\alpha_{f}(\mathrm{deg})$ & 0.057 & 0.031 & 0 \\
$\beta_{f}(\mathrm{deg})$ & 0 & 0 & 0 \\
$\gamma_{f}(\mathrm{deg})$ & -2.815 & 0.026 & -0.094 \\
$\omega_{z f}(\mathrm{deg} / \mathrm{s})$ & 0 & 0 & 0 \\
$\omega_{y f}(\mathrm{deg} / \mathrm{s})$ & 0 & 0 & 0 \\
$\omega_{x f}(\mathrm{deg} / \mathrm{s})$ & 0.861 & 0 & 0 \\
$|\alpha|_{\max }(\mathrm{deg})$ & 10.86 & 9.12 & 9.12 \\
$\left|\beta_{\max }\right|(\mathrm{deg})$ & 1.44 & 1.09 & 0.67 \\
$\left|\gamma_{\max }\right|(\mathrm{deg})$ & 32.61 & 32.61 & 32.61 \\
$\left|\omega_{z \max }\right|(\mathrm{deg} / \mathrm{s})$ & 11.82 & 5.11 & 5.11 \\
$\left|\omega_{y \max }\right|(\mathrm{deg} / \mathrm{s})$ & 1.69 & 1.12 & 0.38 \\
$\left|\omega_{x \max }\right|(\mathrm{deg} / \mathrm{s})$ & 22.41 & 22.41 & 22.41 \\
$J$ & 13478 & 12479 & 9998 \\
\hline
\end{tabular}

\section{Acknowledgments}

This work was supported by the National Natural Science Foundation of China under Grant no. 71501184.

\section{References}

[1] L. Fu, J. Liu, G. Meng, and D. Wang, "Survey of manned/ unmanned air combat decision technology," in Proceedings of the 27th Chinese Control and Decision Conference (CCDC '15), pp. 353-357, IEEE, Qingdao, China, May 2015.

[2] P. T. Jardine, S. Givigi, and A. Noureldin, "Incorporating feedback predictions for optimized UAV attack mission planning," in Proceedings of the 23rd Mediterranean Conference on Control and Automation (MED '15), pp. 740-746, Torremolinos, Spain, June 2015.

[3] X.-X. Hu, Y. Chen, and H. Luo, "Robust decision making for UAV air-to-ground attack under severe uncertainty," Journal of Central South University, vol. 22, no. 11, pp. 4263-4273, 2015.

[4] A. Zhang and F. Guo, "Dynamic task allocation for formation air-to-ground attack," in Proceedings of the 6th International Conference on Advanced Computational Intelligence (ICACI '13), pp. 119-123, IEEE, Hangzhou, China, October 2013.

[5] H. Wang, Z. Guan, and L. Yu, "The application of proportional navigation in the process of UAV air combat guidance and optimization of proportional parameter," in Proceedings of the 33rd Chinese Control Conference (CCC '14), pp. 7892-7896, IEEE, Nanjing, China, July 2014.

[6] P. Zhang, Y. W. Fang, and C. Jin, "A new real-time air-to-air attack zone calculation method," Journal of Ballistic Trajectory, vol. 22, no. 4, pp. 11-18, 2010.

[7] S. Erkan, M. Kandemir, and G. Giger, "Advanced task assignment for unmanned combat aerial vehicles targeting cost efficiency and survivability," in Proceedings of the 46th AIAA Aerospace Sciences Meeting and Exhibit, Reno, Nev, USA, January 2008.

[8] Y. Hui, Y. Nan, S. Chen, Q. Ding, and S. Wu, "Dynamic attack zone of air-to-air missile after being launched in random wind field," Chinese Journal of Aeronautics, vol. 28, no. 5, pp. 15191528, 2015.
[9] D. Alkaher and A. Moshaiov, "Dynamic-escape-zone to avoid energy-bleeding coasting missile," Journal of Guidance, Control, and Dynamics, vol. 38, no. 10, pp. 1908-1921, 2015.

[10] X. Liang, M. Hou, and G. Duan, "Adaptive dynamic surface control for integrated missile guidance and autopilot in the presence of input saturation," Journal of Aerospace Engineering, vol. 28, no. 5, Article ID 04014121, 2015.

[11] S. Shamaghdari, S. K. Nikravesh, and M. Haeri, "Integrated guidance and control of elastic flight vehicle based on robust MPC," International Journal of Robust and Nonlinear Control, vol. 25, no. 15, pp. 2608-2630, 2015.

[12] H. Song, T. Zhang, G. Zhang, and C. Lu, "Integrated interceptor guidance and control with prescribed performance," International Journal of Robust and Nonlinear Control, vol. 25, no. 16, pp. 3179-3194, 2015.

[13] Y. Han, Z. Zheng, and Y. Chong, "Integrated guidance and control design for guided bomb with terminal angle constraint," in Proceedings of the IEEE International Conference on Information and Automation (ICIA '15), pp. 1344-1348, Lijiang, China, August 2015.

[14] H. Song and T. Zhang, "Fast robust integrated guidance and control design of interceptors," IEEE Transactions on Control Systems Technology, vol. 24, no. 1, pp. 349-356, 2016.

[15] Z. Cong and W. Yun-Jie, "Non-singular terminal dynamic surface control based integrated guidance and control design and simulation," ISA Transactions, vol. 63, no. 7, pp. 112-120, 2016.

[16] C. H. Tournes and Y. B. Shtessel, "Integrated guidance and autopilot for dual controlled missiles using higher order sliding mode controllers and observers," in Proceedings of the AIAA Guidance, Navigation and Control Conference and Exhibit, August 2008.

[17] Y. B. Shtessel and C. H. Tournes, "Integrated higher-order sliding mode guidance and autopilot for dual-control missiles," Journal of Guidance, Control, and Dynamics, vol. 32, no. 1, pp. 79-94, 2009.

[18] Y. Wei, M. Z. Hou, and G. R. Duan, "Adaptive multiple sliding surface control for integrated missile guidance and autopilot with terminal angular constraint," in Proceedings of the 29th Chinese Control Conference, pp. 2162-2166, IEEE, Beijing, China, July 2010.

[19] K. Chen, B. Fu, Y. Ding, and J. Yan, "Integrated guidance and control method for the interception of maneuvering hypersonic vehicle based on high order sliding mode approach," Mathematical Problems in Engineering, vol. 2015, Article ID 648231, 19 pages, 2015.

[20] C. Guo and X.-G. Liang, "Integrated guidance and control based on block backstepping sliding mode and dynamic control allocation," Proceedings of the Institution of Mechanical Engineers, Part G: Journal of Aerospace Engineering, vol. 229, no. 9, pp. 1559-1574, 2015.

[21] L. Wen, L. Gao, X. Li, and L. Zhang, "Free pattern search for global optimization," Applied Soft Computing Journal, vol. 13, no. 9, pp. 3853-3863, 2013.

[22] A. F. Ali and M. A. Tawhid, "Hybrid simulated annealing and pattern search method for solving minimax and integer programming problems," Pacific Journal of Optimization, vol. 12, no. 1, pp. 151-184, 2016.

[23] X. Zhao, W. Lin, J. Hao, X. Zuo, and J. Yuan, "Clustering and pattern search for enhancing particle swarm optimization with Euclidean spatial neighborhood search," Neurocomputing, vol. 171, pp. 966-981, 2016. 
[24] F. Kang, J. Li, and H. Li, "Artificial bee colony algorithm and pattern search hybridized for global optimization," Applied Soft Computing Journal, vol. 13, no. 4, pp. 1781-1791, 2013.

[25] F. Nagina, M. Saeed, M. F. Tabassum et al., "Solution of quarter car model by pattern search methods," Science International, vol. 28, no. 1, pp. 7-13, 2016.

[26] S.-J. Choi, D. Lee, I.-K. Kim et al., “Trajectory optimization for a lunar orbiter using a pattern search method," Proceedings of the Institution of Mechanical Engineers, Part G: Journal of Aerospace Engineering, 2016.

[27] H. Li, P. Shi, D. Yao, and L. Wu, "Observer-based adaptive sliding mode control for nonlinear Markovian jump systems," Automatica, vol. 64, pp. 133-142, 2016.

[28] M. Roopaei, M. Zolghadri, and S. Meshksar, "Enhanced adaptive fuzzy sliding mode control for uncertain nonlinear systems," Communications in Nonlinear Science and Numerical Simulation, vol. 14, no. 9-10, pp. 3670-3681, 2009.

[29] M. Furat and İ. Eker, "Chattering-eliminated adaptive slidingmode control: an experimental comparison study," Turkish Journal Of Electrical Engineering \& Computer Sciences, vol. 24, pp. 605-620, 2016.

[30] H.-L. Xin, J.-B. Hu, and P. Gao, "New gain-scheduling variable structure tracking control for a class of nonlinear systems," Systems Engineering and Electronics, vol. 33, no. 6, pp. 13621366, 2011. 


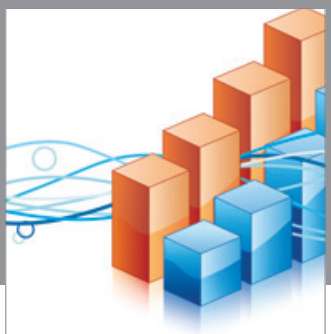

Advances in

Operations Research

vatem alat4

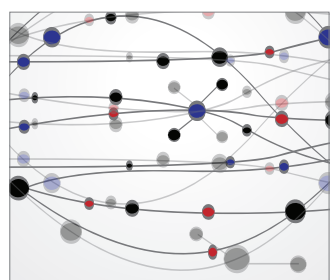

\section{The Scientific} World Journal
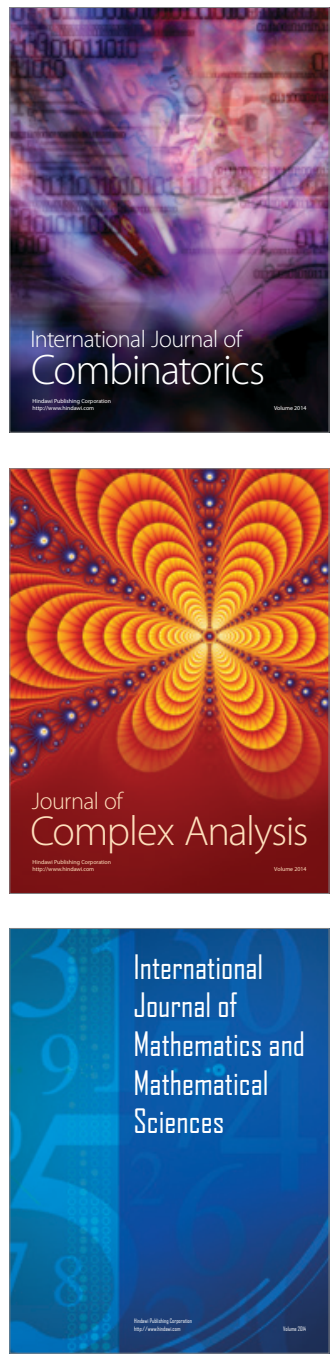
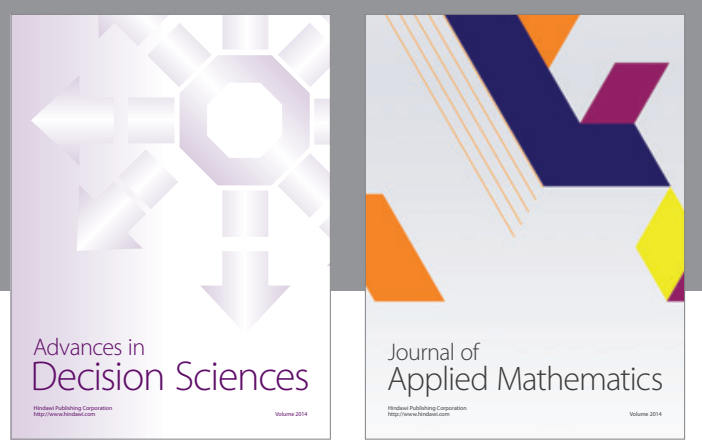

Algebra

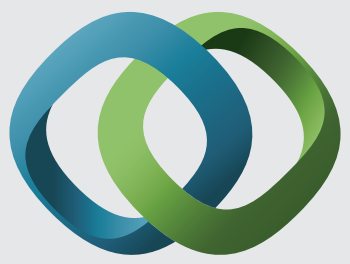

\section{Hindawi}

Submit your manuscripts at

http://www.hindawi.com
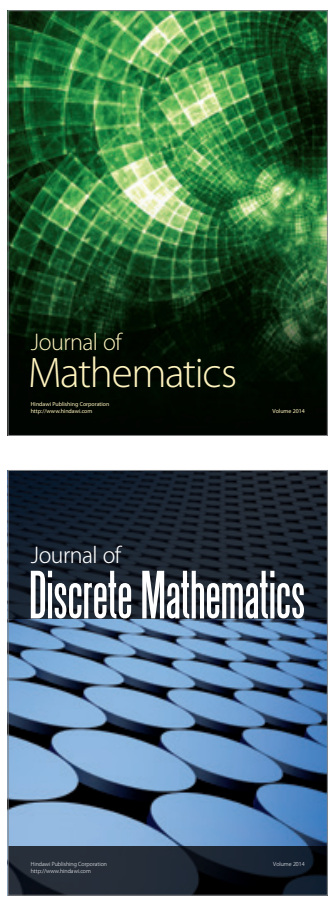

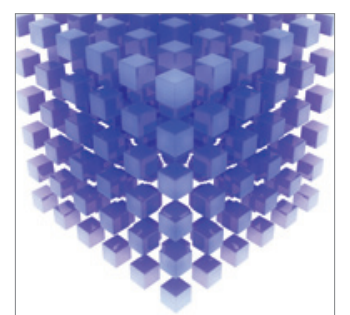

Mathematical Problems in Engineering
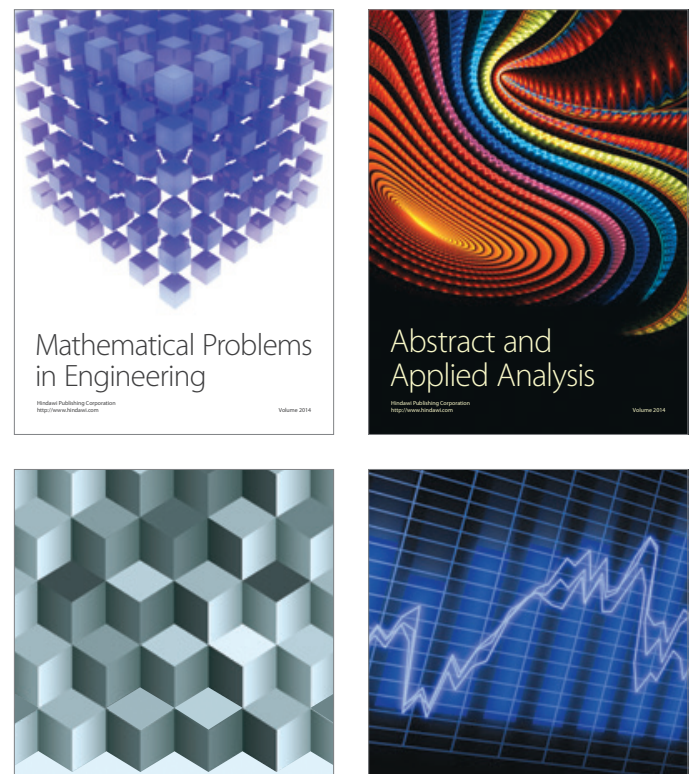

Journal of

Function Spaces

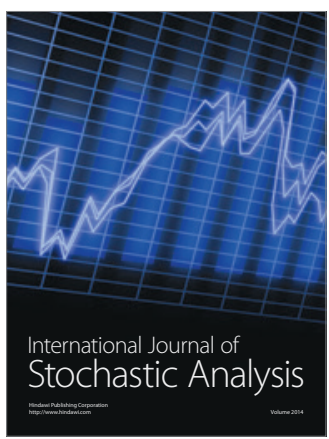

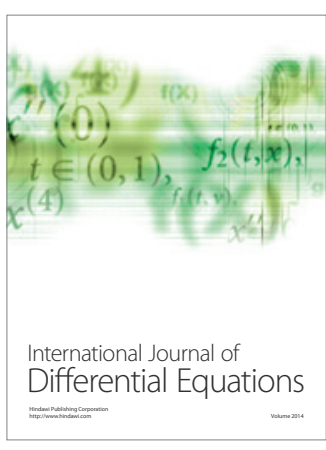
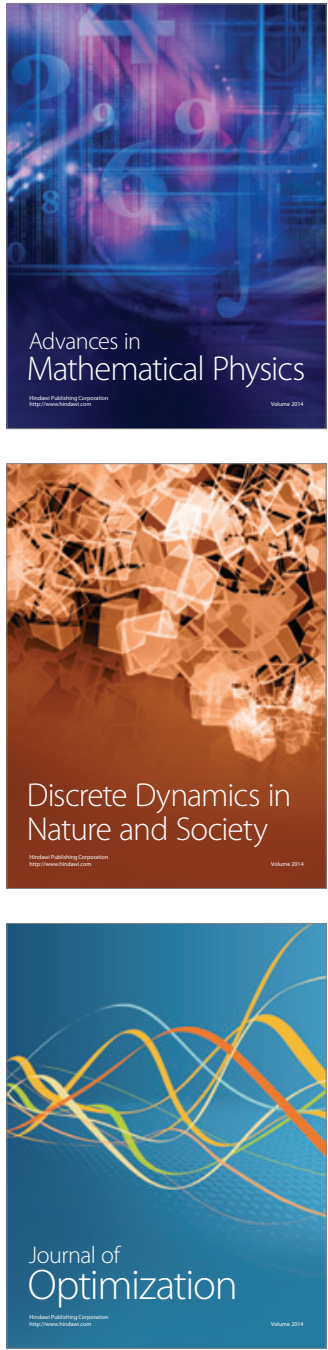